\title{
A fixed-point characterization of the optimal costate in finite-horizon optimal control problems
}

\author{
M. Sassano, Senior Member, IEEE and A. Astolfi, Fellow, IEEE
}

\begin{abstract}
A fixed-point characterization of the optimal costate in finite-horizon optimal control problems for nonlinear systems is presented. It is shown that the optimal initial condition of the costate variable must be a fixedpoint, for any time, of the composition of the forward and backward flows of the underlying Hamiltonian dynamics. Such an abstract property is then translated into a constructive condition by relying on a sequence of repeated Lie brackets involving the Hamiltonian dynamics and evaluated at a single point in the state-space. This leads to a system of algebraic equations in the unknown initial optimal costate that allows achieving a desired degree of accuracy of the approximation while always consisting of a number of equations equal to the dimension of the state of the underlying system, regardless of the achieved accuracy. A dual characterization of the optimal terminal value of the state is also discussed, together with a few computational aspects of the proposed strategy.
\end{abstract}

\section{INTRODUCTION}

One of the most classical control problem consists in steering the state of the underlying controlled plant from an initial condition - e.g. deriving from a perturbation of a nominal operating condition or from a prescribed shift of the corresponding working point for the system - to a desired final condition or equilibrium point. It is therefore natural that tools and strategies capable of achieving such a control objective in an optimal way would have attracted intensive research interest in the past decades, see e.g. [1], [2], [3], [4], [5], [6], [7]. The above control task can be naturally formulated as an infinite-horizon optimal control problem [8], provided the time available to perform the steering is sufficiently large, compared to the time-scales of the underlying plant. Interestingly, whenever it is instead crucial to complete the control task within a prescribed time interval, fixed a priori, while less attention is paid to the value of the state at the end of such an interval, the structure of the solution to such

This work has been partially supported by the European Union's Horizon 2020 Research and Innovation Programme under grant agreement No 739551 (KIOS CoE), and by Italian Ministry for Research in the framework of the 2017 Program for Research Projects of National Interest (PRIN), Grant no. 2017YKXYXJ.

$M$. Sassano is with the Dipartimento di Ingegneria Civile e Ingegneria Informatica, Università di Roma "Tor Vergata", Via del Politecnico, 1 00133 Roma, Italy (Email: mario.sassano@uniroma2.it).

A. Astolfi is with the Department of Electrical and Electronic Engineering, Imperial College London, London SW7 2AZ, UK and with the Dipartimento di Ingegneria Civile e Ingegneria Informatica, Università di Roma "Tor Vergata", Via del Politecnico, 100133 Roma, Italy (Email: a.astolfi@ic.ac.uk). a seemingly similar problem becomes notably different, being for instance intrinsically time-varying [5].

Despite such differences two main strategies, common to the so-called infinite-horizon and finite-horizon optimal control problems, have been introduced and deeply explored in the second half of the last century, i.e. Dynamic Programming (DP), see e.g. [2], [9], and Pontryagin's Minimum (or Maximum) Principle (PMP) [10].

The former approach characterizes the optimal feedback in terms of the solution to a certain quadratic partial differential equation (PDE), i.e. the so-called Hamilton-Jacobi-Bellman (HJB) equation [4]. Methods inspired by DP are particularly appealing since they provide necessary and sufficient conditions for optimality and permit the characterization of the optimal solution, as well as of the optimal cost, for any initial condition in the state-space. In the case of infinite-horizon control problems strategies to approximate the optimal control law have been envisioned, see e.g. [11], [12], [13], [14], [15], [16], [17], [18]. Along a different line to circumvent the computational issues arising in the solution of the HJB equation, an alternative (weaker) notion of solution has been proposed in the case of non-differentiable value functions, see e.g. [19], [20], introducing the notion of viscosity solution of the HJB equation. However, in the case of finite-horizon optimal control problems the task of determining a closedform expression for the solution to the HJB PDE is further complicated by the intrinsic dependence of the value function on time.

On the other hand, methods based on the PMP provide - without additional conditions, such as convexity - only necessary conditions for optimality. As a consequence such strategies may be employed only to identify candidate optimal solutions or extremals. Moreover, the characterization of the optimal control law heavily relies on the knowledge of the initial condition of the state of the system even for infinitehorizon control problems, hence essentially leading to openloop strategies. Nonetheless, the wide-spread use of such strategies is essentially motivated by the simplicity of the underlying conditions, provided in terms of ordinary differential equations (instead of partial differential equations) that should be satisfied by the optimal process together with an auxiliary variable (the costate). While in the case of infinitehorizon problems determining the correct boundary conditions for such a dynamical system essentially requires to compute an explicit solution to the HJB PDE, hence recovering the computational complexity of the DP approach, in the case of finite horizon optimal control problems it can be recast in 
terms of a two-point boundary value problem (TPBVP) for a nonlinear system.

As a consequence, intensive research efforts have been devoted to the design of efficient strategies to determine or approximate the solution to TPBVPs for linear or nonlinear systems. Indeed, by relying on the increasingly growing computational capabilities available in modern applications, the so-called computational optimal control has become a separate field of active research [21], [22], [23], [24], [25], [26], [27], [28]. Such efforts resulted in the development of several efficient tools and software devoted to the solution to TPBVPs, including e.g. GPOPS [29], ICLOCS2 [30], as well as the approaches in [31], [32], [33], [34], [35] among several others. Although diverse, the above methods share common features: they are based on a direct transcription of the underlying continuous-time optimal control problem into a nonlinear programming problem (NLP), which can be then solved with standard [36] or more dedicated approaches, e.g. IPOPT [37]. In particular, direct collocation methods rely on a discretization of the state and control at a collection of suitably defined points within the time interval of interest. In $h$-methods, the time interval is divided into a prescribed grid and the state is approximated by means of polynomials of fixed degree in each cell of the mesh, with convergence to the optimal solution achieved by increasing the number of collocated points. On the other hand, in $p$-methods convergence to the optimal solution is obtained by increasing the order of the polynomial. Recently, $h p$-methods [21] that combine the two previous approaches adaptively adjusting the dimension of the grid and the degree of the polynomial have been envisioned. As a common feature to all the previous approaches, the transcription of the continuous-time optimal control problem yields a sparse, although typically very large, NLP problem, hence a very large system of nonlinear algebraic equations.

The main contribution of this paper consists in proposing a design strategy to compute or approximate the optimal initial condition for the costate variable in finite-horizon optimal control problems by solving a system of $n$ algebraic equations, regardless of the prescribed degree of accuracy. The result is achieved by first discussing a fixed-point characterization of the optimal costate, which is then translated into more constructive conditions by relying on a sequence of Lie brackets. These involve the underlying Hamiltonian vector field together with a suitably defined projective vector field. Such Lie brackets are then evaluated at the initial condition of the state variable, thus leading to a system of algebraic equations in the (unknown) costate initial condition. An identical strategy is then extended to the computation of the optimal terminal value of the state variable. Somewhat similar ideas are employed in [38] to construct solutions to infinite horizon optimal control problems. In particular, therein the key aspect consists in considering the composition of the flows of the (projected) Hamiltonian dynamics and that of the closed-loop optimal system in the original coordinates. Note that an identical strategy could not be straight-forwardly pursued in the context of finite horizon problems, since the latter vector field becomes time-varying, hence introducing challenges that can be only circumvented by considering instead the composition of the forward and backward Hamiltonian system, as pursued herein. The rest of the paper is organized as follows. The definition of the finite-horizon optimal control problem dealt with is provided in Section II together with additional results and assumptions instrumental for the subsequent derivations. The main statements - consisting of a design strategy that allows to characterize or approximate the optimal initial condition of the costate variable - are presented in Section III. The specialization to the linear setting of the above results is instead the topic of Section IV, while the objective of Section V is to extend the previous machinery to compute (or approximate) also the value of the state variable at the end of the interval of interest, which is unknown, similarly to the costate initial condition. Finally, the paper is concluded by extensive discussions on several computational aspects of the approach in Section VI and further comments in Section VII.

Notation. $\mathbb{N}$ defines the set of positive integers, $\mathbb{N}_{0}=$ $\mathbb{N} \cup\{0\}$, and $\mathbb{R}_{\geqslant 0}\left(\mathbb{R}_{>0}\right)$ defines the set of non-negative (positive) real numbers. $\sigma(A)$ denotes the spectrum of the matrix $A \in \mathbb{R}^{n \times n}$. Given a symmetric matrix $M=M^{\top}$, $M \succeq 0$, denotes a positive semi-definite matrix, namely such that $v^{\top} M v \geqslant 0$ for any vector $v$; similarly $M \succ 0$ denotes a positive definite matrix. $I_{n}$ denotes the identity matrix of dimension $n$. Given two vectors $x_{1} \in \mathbb{R}^{n_{1}}$ and $x_{2} \in \mathbb{R}^{n_{2}},\left(x_{1}, x_{2}\right)$ denotes the vector $\left[\begin{array}{ll}x_{1}^{\top} & x_{2}^{\top}\end{array}\right]^{\top}$. For a continuously differentiable function $f: \mathbb{R}^{n} \rightarrow \mathbb{R}, \nabla_{x} f$ denotes the column vector of the partial derivatives of $f$, namely $\nabla_{x} f=\left[\partial f(x) / \partial x_{1}, \ldots, \quad \partial f(x) / \partial x_{n}\right]^{\top}$. Given two vector fields $f: \mathbb{R}^{n} \rightarrow \mathbb{R}^{n}$ and $g: \mathbb{R}^{n} \rightarrow \mathbb{R}^{n}$, the Lie bracket of $f$ and $g$, denoted $[f, g](x)$, is defined as $[f, g](x)=\left(\nabla_{x} g\right) f(x)-\left(\nabla_{x} f\right) g(x)$. The repeated Lie bracket operation of a vector field $g$ with the same vector field $f$ is recursively defined as $\operatorname{ad}_{f}^{k} g(x)=\left[f, \operatorname{ad}_{f}^{k-1} g\right](x)$, with $\operatorname{ad}_{f}^{0} g(x)=g(x)$. Given an ordinary differential equation $\dot{x}=f(x)$ with boundary condition $x(0)=x_{0}$ and the vector field $f: \mathbb{R}^{n} \rightarrow \mathbb{R}^{n}$ sufficiently smooth, the mapping $\varphi_{f}: \mathbb{R} \times \mathbb{R}^{n} \rightarrow \mathbb{R}^{n}$ denotes the corresponding flow such that $\partial \varphi_{f}\left(t ; x_{0}\right) / \partial t=f\left(\varphi_{f}\left(t ; x_{0}\right)\right)$ for any $t \geqslant 0$ and $x_{0}$. The notation $\mathcal{C}^{\kappa}$ indicates the space of functions that possess continuous derivatives of order less than or equal to $\kappa$. A function $f: \mathcal{X} \rightarrow \mathbb{R}^{n}$ is analytic in the open set $\mathcal{X}$, denoted as $f \in \mathcal{C}^{\omega}(\mathcal{X})$, if for every $v \in \mathcal{X}$ there is a neighborhood $\mathcal{U}$ such that the Taylor series expansion centered at $v$ converges pointwise to $f(x)$ for $x \in \mathcal{U}$. Given a function $f: \mathcal{X} \rightarrow \mathcal{X}$, a point $c$ in the topological space $\mathcal{X}$ is a fixed-point of $f$ if $f(c)=c$, namely if $(c, c) \in$ graph $f . \mathbb{B}(\bar{x}, R)$ denotes the open ball of radius $R>0$ centered at $\bar{x} \in \mathbb{R}^{n}$, i.e. $\mathbb{B}(\bar{x}, R):=\left\{x \in \mathbb{R}^{n}:|x-\bar{x}|<R\right\}$, whereas $\bar{B}(\bar{x}, R)$ denotes the closure of $\mathbb{B}(\bar{x}, R)$.

\section{Preliminaries And PROBlem Statement}

Consider an optimal control problem defined over a finite horizon and described by

$$
\begin{aligned}
\min _{u} J_{t_{0}, x_{0}}(u): & =\frac{1}{2} \int_{t_{0}}^{T}\left(q(x(\tau))+\|u(\tau)\|^{2}\right) d \tau, \\
\text { s.t. } \dot{x}(t) & =f(x(t))+g(x(t)) u(t), \quad x\left(t_{0}\right)=x_{0}
\end{aligned}
$$


with $x(t) \in \mathcal{X} \subseteq \mathbb{R}^{n}$, where $\mathcal{X}$ is a $\mathrm{n}$-dimensional smooth manifold, and $u(t) \in \mathbb{R}^{m}, u \in \mathcal{C}^{0}([0, T])$, while $x_{0} \in \mathcal{X}$ is a given initial condition of the differential equation (1b). It is worth observing that - even in the absence of additional requirements, e.g. hard constraints (saturation) on the control input - the optimal control problem formulated in (1a)-(1b) already exhibits the features and computational challenges that are addressed in this paper. In fact - as anticipated herein and briefly recalled in the rest of this section - it is well known that the problem (1a)-(1b) may be tackled either by relying on Pontryagin's Minimum Principle or on a Dynamic Programming approach, which however requires the computation of the explicit solution of the Hamilton-Jacobi-Bellman partial differential equation. While the latter can be seldom determined in practice, the former strategy yields typically a two-point boundary value problem defined with respect to the underlying (nonlinear) Hamiltonian dynamics. To address such a problem several methods have been proposed, including the so-called collocation methods [23] and shooting methods [39].

The main objective of this paper is to propose an approach alternative to the above mentioned methods, which hinges upon more abstract, geometric, properties of the optimal costate and for which - differently from the former methods - the number of algebraic equations does not grow with increasing accuracy of the approximation and remains equal to the dimension of the state. On the other hand, instead of considering the entire forward flow of an unstable system as in the latter strategies, herein the required conditions are obtained by characterizing the optimal costate by means of subsequent Lie brackets evaluated at a single time instant.

Assumption 1: The function $q: \mathbb{R}^{n} \rightarrow \mathbb{R}_{>0}, q(0)=0$, and the vector fields $f: \mathbb{R}^{n} \rightarrow \mathbb{R}^{n}, f(0)=0$, and $g_{i}: \mathbb{R}^{n} \rightarrow \mathbb{R}^{n}$, $g_{i}(0)=0, i=1, \ldots, m$, are analytic at $x=0$ with radius of convergence $r_{x}$ such that $\mathcal{X} \subset \mathbb{B}\left(0, r_{x}\right)$.

The function $V^{\star}: \mathbb{R}_{\geqslant 0} \times \mathcal{X} \rightarrow \mathbb{R}_{\geqslant 0}$ defined as $V^{\star}\left(t_{0}, x_{0}\right)=$ $\min _{u}\left\{J_{t_{0}, x_{0}}(u)\right\}$ is referred to as the value function of the problem (1). Since the cost functional (1a) and the vector fields appearing in (1b) do not depend explicitly on the time $t$, in the following it is assumed that $t_{0}=0$ without loss of generality.

Remark 1: The presence of a terminal cost, $m(x(T))$, with $m: \mathcal{X} \rightarrow \mathbb{R}_{\geqslant 0}$ a real analytic function, on the state of the system (1b) in (1a) can be straight-forwardly encompassed into the framework of (1a) by replacing the terminal cost with an equivalent Lagrangian cost. In fact, since

$$
m(x(T))=m\left(x_{0}\right)+\int_{0}^{T} \frac{d}{d t} m(x(t)) d t
$$

and since $x(0)=x_{0}$ is a constant with respect to $u$, the terminal cost $m$ can be included in the Lagrangian term by defining the modified running cost $\tilde{q}(x, u)=q(x)+$ $2\left(\nabla_{x} m(x)\right)(f(x)+g(x) u)$, provided $\tilde{q}(x, u)+\|u\|^{2}$ remains positive definite with respect to $x$ and $u$ for consistency with the definition of (1a).

Consider the underlying (minimized) Hamiltonian function $\mathbf{H}: T^{\star} \mathcal{X} \rightarrow \mathbb{R}$, with $T^{\star} \mathcal{X}$ denoting the cotangent bundle over
$\mathcal{X}$ with natural coordinates $(x, \lambda)$, defined as

$$
\mathbf{H}(x, \lambda):=\frac{1}{2} q(x)+\lambda^{\top} f(x)-\frac{1}{2} \lambda^{\top} g(x) g(x)^{\top} \lambda .
$$

The optimal process $\left(x^{\star}(t), \lambda^{\star}(t)\right)$ then satisfies the Hamiltonian dynamics

$$
\left[\begin{array}{c}
\dot{x} \\
\dot{\lambda}
\end{array}\right]=J \nabla \mathbf{H}(x, \lambda):=X_{\mathbf{H}}(x, \lambda),
$$

with

$$
J:=\left[\begin{array}{rr}
0 & I_{n} \\
-I_{n} & 0
\end{array}\right]
$$

and $X_{\mathbf{H}}$ denoting the Hamiltonian vector field, together with the split boundary conditions $(x(0), \lambda(T))=\left(x_{0}, 0\right)$. Alternatively, the Hamiltonian dynamics (4) can be initialized at $(x(0), \lambda(0))=\left(x_{0}, \nabla_{x} V^{\star}\left(t_{0}, x_{0}\right)\right)$, where the value function $V^{\star}(t, x), V^{\star}: \mathbb{R} \times \mathcal{X} \rightarrow \mathbb{R}_{>0}$, satisfies the Hamilton-JacobiBellman partial differential equation

$$
-\frac{\partial V^{\star}}{\partial t}=\frac{1}{2} q(x)+\frac{\partial V^{\star}}{\partial x} f(x)-\frac{\partial V^{\star}}{\partial x} g(x) g(x)^{\top}\left(\frac{\partial V^{\star}}{\partial x}\right)^{\top}
$$

for all $x \in \mathcal{X}$ and $t \in[0, T]$, together with the boundary condition $V^{\star}(T, x)=0$ for any $x \in \mathcal{X}$. As a consequence, the computation of the value function constitutes a critical bottleneck for the solution of problem (1) regardless if one relies on the DP strategy or on PMP approach, since for any $x_{0} \in \mathcal{X}$ the costate variable $\lambda(0)$ should be initialized at $\lambda_{0}^{\star}\left(x_{0}\right):=$ $\nabla_{x} V^{\star}\left(0, x_{0}\right)$.

In the following it is assumed that, for given initial condition $x_{0}$ and terminal time $T>0$, the optimal control problem (1) admits a solution that satisfies additional continuity properties, as detailed in the statement below.

Assumption 2: Given an initial condition $x_{0} \in \mathcal{X}$ and a terminal time $T \in \mathbb{R}_{>0}$, there exists a unique smooth solution $V^{\star}: \mathbb{R} \times \mathcal{X} \rightarrow \mathbb{R}_{>0}$ of (6), for $t \in[0, T]$ and for all $x \in \mathcal{X}$ together with the boundary condition $V^{\star}(T, x)=0$ for all $x \in \mathcal{X}$.

Finally, introducing $z:=(x, \lambda)$ and considering the Hamiltonian dynamics $\dot{z}=X_{\mathbf{H}}(z), z_{0}=\left(x_{0}, \lambda_{0}\right)$, let $\Phi_{t}^{X_{\mathbf{H}}}$ : $\mathcal{X} \times \mathbb{R}^{n} \rightarrow \mathcal{X} \times \mathbb{R}^{n}$ denote the one-parameter group of local diffeomorphisms, under the operation of composition, that defines an integral curve of $X_{\mathbf{H}}$ for any $z \in T^{\star} \mathcal{X}$, namely $\Phi_{t}^{X_{\mathbf{H}}}(z)=\varphi_{X_{\mathbf{H}}}\left(t ; x_{0}, \lambda_{0}\right)$, where $\varphi_{X_{\mathbf{H}}}: \mathbb{R}_{\geqslant 0} \times \mathcal{X} \times \mathbb{R}^{n} \rightarrow \mathcal{X} \times$ $\mathbb{R}^{n}$ defines the flow of the vector field $X_{\mathbf{H}}$. As a consequence of Assumption 2 the flow $\varphi_{X_{\mathbf{H}}}\left(t ; x_{0}, \nabla_{x} V^{\star}\left(0, x_{0}\right)\right)$ is defined for all $t \in[0, T]$ and it satisfies $\lambda(t)=\nabla_{x} V^{\star}(t, x(t))$, see for instance [10, Chapter 1]. Moreover, as a result of Assumption 1, the costate $\lambda: \mathbb{R} \rightarrow \mathbb{R}^{n}$ is an analytic function of time. Suppose, in addition, that the following requirement is satisfied on the radius of convergence for the analyticity property of the costate $\lambda$ with respect to time.

Assumption 3: The flow $\varphi_{X_{\mathbf{H}}}\left(t ; x_{0}, \lambda_{0}^{\star}\left(x_{0}\right)\right)$ is an analytic function at $t=0$ with the property that its radius of convergence $r_{H}$ satisfies $r_{H}>2 T$. 


\section{A FIXED-POINT CHARACTERIZATION OF THE OPTIMAL COSTATE}

Let $\pi: T^{\star} \mathcal{X} \rightarrow \mathcal{X}$ denote the natural projection operator that maps an element of $T_{x}^{\star} \mathcal{X}$ into the corresponding base point $x \in \mathcal{X}$. The following statement provides a fixed-point characterization of the optimal costate variable.

Theorem 1: Consider the finite-horizon optimal control problem (1). Let $x_{0} \in \mathcal{X}$ and $T \in \mathbb{R}_{>0}$ be given and suppose that Assumptions 1-3 hold. Then a vector $\lambda_{0} \in \mathbb{R}^{n}$ is such that $\lambda_{0}=\nabla_{x} V^{\star}\left(0, x_{0}\right)$ if and only if there exists a function $\lambda_{a}:[0, T] \rightarrow \mathbb{R}^{n}$, analytic at $t=0$, with radius of convergence larger than $T$ and such that $\lambda_{a}(T)=0$, with the property that

$$
\omega(t):=\varphi_{X_{\mathbf{H}}}\left(-t ; \pi \circ \varphi_{X_{\mathbf{H}}}\left(t ; x_{0}, \lambda_{0}\right), \lambda_{a}(t)\right)=\left(x_{0}, \lambda_{0}\right),
$$

for all $t \in[0, T]$.

Proof: $(\Rightarrow)$ Since the function $t \mapsto V^{\star}(t, x(t))$ is smooth, hence at least twice continuously differentiable, it follows that the trajectory $x: \mathbb{R} \rightarrow \mathbb{R}^{n}$ yields the optimal timeevolution of the state variable only if it can be obtained as the projection on the $x$-coordinates of $a$ trajectory of the Hamiltonian dynamics (4) and the costate variable verifies $\lambda(t)=\nabla_{x} V^{\star}(t, x(t))$, for all $t \in[0, T]$, which is analytic by Assumptions 1 and 2. Moreover, recalling that the value function $V^{\star}(t, x), V^{\star}: \mathbb{R} \times \mathcal{X} \rightarrow \mathbb{R}_{>0}$, satisfies the HamiltonJacobi-Bellman partial differential equation (6) for all $x \in$ $\mathcal{X}$ and $t \in[0, T]$, together with the boundary condition $V^{\star}(T, x)=0$ for all $x \in \mathcal{X}$, it follows that $\nabla_{x} V^{\star}(T, x)=0$ for any $x \in \mathcal{X}$. Therefore, $\lambda_{0}=\nabla_{x} V^{\star}\left(0, x_{0}\right)$ verifies (7) together with the feasible selection $\lambda_{a}(\cdot):=\nabla_{x} V^{\star}(\cdot, x(\cdot))$.

$(\Leftarrow)$ For any $x_{0} \in \mathcal{X} \subset \mathbb{R}^{n}, \lambda_{0}$ yields the optimal initial condition of the costate variable if the composition of the forward flow (for $t$ seconds) along the vector field $X_{\mathbf{H}}$ from $\left(x_{0}, \lambda_{0}\right)$ with the backward flow (for $t$ seconds) along the same vector field - considering the state component reached at time $t$ and the costate component potentially set to the value of an analytic function $\lambda_{a}(t)$ - yields again $\left(x_{0}, \lambda_{0}\right)$, for all $t \geqslant 0$. Since the latter task alone may be accomplished by letting $\lambda_{a}(\cdot)$ be defined as any function $v(x(\cdot))$ such that the manifold $\left\{(x, \lambda) \in \mathcal{X} \times \mathbb{R}^{n}: \lambda=v(x)\right\}$ is invariant for (4), namely any $v: \mathcal{X} \rightarrow \mathbb{R}^{n}$ with the property that $\left(\frac{\partial v}{\partial x} \nabla_{\lambda} \mathbf{H}(x, \lambda)+\right.$ $\left.\nabla_{x} \mathbf{H}(x, \lambda)\right)\left.\right|_{\lambda=v(x)}=0$, for any $x \in \mathcal{X}$, sufficiency of (7) follows by additionally requiring that $\lambda_{a}(T)=0$ and by uniqueness of the trajectories of (4).

Remark 2: The condition (7) entails that the mapping $\left(x_{0}, \lambda_{0}\right) \mapsto \varphi_{X_{\mathbf{H}}}\left(-t ; \pi \circ \varphi_{X_{\mathbf{H}}}\left(t ; x_{0}, \lambda_{0}\right), \lambda_{a}(t)\right)$ possesses a fixed-point at $\left(x_{0}, \nabla_{x} V^{\star}\left(0, x_{0}\right)\right)$ for all $t \geqslant 0$, obtained by letting $\lambda_{a}(t)=\nabla_{x} V^{\star}\left(t, x^{\star}(t)\right)$. Therefore, the operations of flowing along the vector field $X_{\mathbf{H}}$ forward for $t$ seconds, projecting the reached composite state into $\mathcal{X}$, and flowing backward again along $X_{\mathbf{H}}$ for $t$ seconds must yield the initial condition $\left(x_{0}, \lambda_{0}\right)$ of the extended system, provided the costate component of $X_{\mathbf{H}}$ is suitably set to $\lambda_{a}(t)$ at time $t$.

Let now id : $\mathcal{X} \times \mathbb{R}^{n} \rightarrow \mathcal{X} \times \mathbb{R}^{n}$ denote the identity function, namely $\operatorname{id}(z)=z$, and define the partial projective mapping

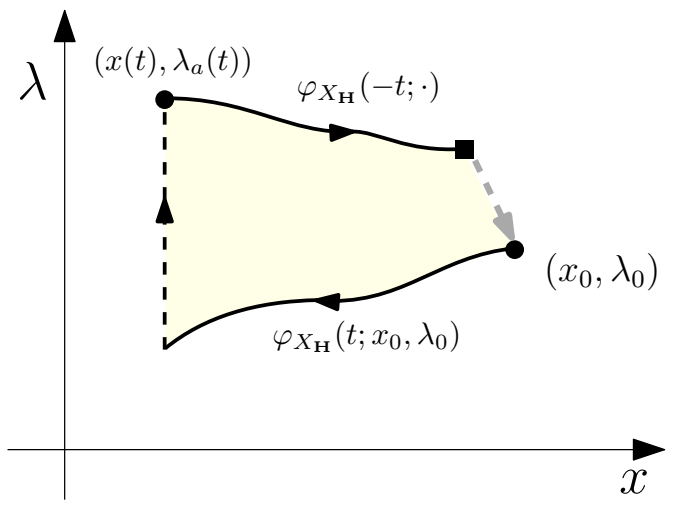

Fig. 1. Graphical representation of the composition of the forward and backward flows of the Hamiltonian dynamics (4). The gray dashed vector describes the distance of $\left(\boldsymbol{x}_{0}, \boldsymbol{\lambda}_{0}\right)$ from being a fixed-point of the composition in (7).

$p: \mathcal{X} \times \mathbb{R}^{n} \rightarrow \mathcal{X} \times \mathbb{R}^{n}$ as

$$
p(z)=\left[\begin{array}{l}
\pi \circ z \\
h(z)
\end{array}\right],
$$

where $h: \mathcal{X} \times \mathbb{R}^{n} \rightarrow \mathbb{R}^{n}$ is an arbitrary analytic function. Suppose that the following standing assumption holds.

Assumption 4: There exist positive constants $c_{\ell} \in \mathbb{R}_{>0}$ and $c_{u} \in \mathbb{R}_{>0}$ such that

$$
\begin{aligned}
& \inf _{t \in[0, T], x \in \mathcal{X}} \underline{\sigma}\left(\left(\Phi_{-t}^{X_{\mathbf{H}}}\right)_{\star}\right) \geqslant c_{\ell}, \\
& \sup _{t \in[0, T], x \in \mathcal{X}} \bar{\sigma}\left(\left(\Phi_{-t}^{X_{\mathbf{H}}}\right)_{\star}\right) \leqslant c_{u},
\end{aligned}
$$

with $\left(\Phi_{-t}^{X_{\mathbf{H}}}\right)_{\star}$ denoting the differential of $\Phi_{-t}^{X_{\mathbf{H}}}$.

Note that (9a) in particular implies that the differential of the flow $\Phi_{-t}^{X_{\mathrm{H}}}$ is an invertible matrix for any $t \in[0, T]$.

Theorem 2: Consider the finite-horizon optimal control problem (1). Let $x_{0} \in \mathcal{X}$ and $T \in \mathbb{R}_{>0}$ be given and suppose that Assumptions 1-4 hold. Then a vector $\lambda_{0} \in \mathbb{R}^{n}$ is such that $\lambda_{0}=\nabla_{x} V^{\star}\left(0, x_{0}\right)$ if and only if there exist $\lambda_{k}:=$ $h_{k}\left(x_{0}, \lambda_{0}\right) \in \mathbb{R}^{n}$ with $h_{k}(z):=\left\langle\nabla h_{k-1}, X_{\mathbf{H}}\right\rangle, k=1,2, \ldots$ and $h_{0}(z)=h(z)$, with the properties that

$$
\operatorname{ad}_{X_{\mathbf{H}}}^{k} p\left(x_{0}, \lambda_{0}\right)=\operatorname{ad}_{X_{\mathbf{H}}}^{k} \operatorname{id}\left(x_{0}, \lambda_{0}\right),
$$

for $k=1,2, \ldots$, and $\sum_{k=0}^{\infty} \lambda_{k} \frac{T^{k}}{k !}=0$.

Proof: Since the function $t \mapsto \omega(t)$ defined in the lefthand side of (7) is analytic at $t=0$, the condition on the right-hand side of (7) is equivalent to requiring that $\omega(0)=$ $\left(x_{0}, \lambda_{0}\right)$, which holds for any $\left(x_{0}, \lambda_{0}\right) \in \mathcal{X} \times \mathbb{R}^{n}$, and that $d^{k} \omega(t) /\left.d t^{k}\right|_{t=0}=0$. Define then the analytic function

$$
W(t)=\left(\Phi_{-t}^{X_{\mathbf{H}}}\right)_{\star} p\left(\Phi_{t}^{X_{\mathbf{H}}}\left(z_{0}\right)\right)
$$

with $z_{0}=\left(x_{0}, \lambda_{0}\right)$ and the vector field $p$ introduced in (8), and note that by the Campbell-Baker-Hausdorff formula $W$ : $\mathbb{R} \rightarrow \mathbb{R}^{2 n}$ can be expanded in the form [40]

$$
W(t)=\sum_{k=0}^{\infty} \operatorname{ad}_{X_{\mathbf{H}}}^{k} p\left(z_{0}\right) \frac{t^{k}}{k !},
$$


namely such that

$$
\left.\frac{d^{k} W(t)}{d t^{k}}\right|_{t=0}=\operatorname{ad}_{X_{\mathbf{H}}}^{k} p\left(z_{0}\right)
$$

Moreover, by noting that $\omega$ may be written as $\omega(t)=$ $\Phi_{-t}^{X_{\mathbf{H}}} \circ p\left(\Phi_{t}^{X_{\mathbf{H}}}\left(z_{0}\right)\right)$ and by relying on (9a), it follows that $\left(\Phi_{-t}^{X_{\mathbf{H}}}\right)_{\star}^{-1} W(t)=p\left(\Phi_{t}^{X_{\mathbf{H}}}\left(z_{0}\right)\right)$, hence

$$
\omega(t)=\Phi_{-t}^{X_{\mathbf{H}}}\left(\left(\Phi_{-t}^{X_{\mathbf{H}}}\right)_{\star}^{-1} W(t)\right) .
$$

Therefore, $\omega(t)=\left(x_{0}, \lambda_{0}\right)$ for all $t \geqslant 0$ if and only if $W(t)=$ $\left(\Phi_{-t}^{X_{\mathbf{H}}}\right)_{\star} \Phi_{t}^{X_{\mathbf{H}}}$ for all $t \geqslant 0$, which in turn is equivalent to

$$
\operatorname{ad}_{X_{\mathbf{H}}}^{k} p\left(z_{0}\right)=\left.\left(\frac{d^{k}}{d t^{k}}\left(\Phi_{-t}^{X_{\mathbf{H}}}\right)_{\star} \Phi_{t}^{X_{\mathbf{H}}}\right)\right|_{t=0}
$$

The latter is then equivalent to (10) by applying again the Campbell-Baker-Hausdorff formula with $p$ replaced by the identity function on $\mathcal{X} \times \mathbb{R}^{n}$. The proof is concluded then by noting that $h(z(T))=0$ by the summation requirement on $\lambda_{k}$ and that $\lambda_{a}(t)=h(z(t))=\sum_{k=0}^{\infty} \lambda_{k}\left(t^{k} / k !\right)$.

The conditions in (10) consist of a system of algebraic equations that are linear in the variables $\lambda_{i}, i=1,2, \ldots$, although nonlinear in $\lambda_{0}$. The following statement shows that the conditions (10) can be equivalently recast into a system of $n$ equations in the variable $\lambda_{0}$ alone. To this end, consider first the definition of the operator $\mathcal{D}$, introduced in [38], as follows.

Definition 1: Given a smooth vector field $f: \mathbb{R}^{n} \rightarrow \mathbb{R}^{n}$, consider the operator $\mathcal{D}_{i}(f)$ recursively defined as $\mathcal{D}_{0}(f)=f$ and

$$
\mathcal{D}_{i}(f)=\left(\nabla \mathcal{D}_{i-1}(f)\right) \mathcal{D}_{0}(f)
$$

for $i \in \mathbb{N}$, where $\nabla f$ denotes the Jacobian matrix of the vectorvalued function $\mathrm{f}$.

Theorem 3: Consider the finite-horizon optimal control problem (1). Let $x_{0} \in \mathcal{X}$ and $T \in \mathbb{R}_{>0}$ be given and suppose that Assumptions 1-4 hold. Then a vector $\lambda_{0} \in \mathbb{R}^{n}$ is such that $\lambda_{0}=\nabla_{x} V^{\star}\left(0, x_{0}\right)$ if and only if

$$
\mathcal{T}\left(\lambda_{0}\right):=\lambda_{0}+N \sum_{i=1}^{\infty} \mathcal{D}_{i-1}\left(X_{\mathbf{H}}\left(x_{0}, \lambda_{0}\right)\right) \frac{T^{i}}{i !}=0
$$

with $N:=\left[\begin{array}{cc}0 & I_{n}\end{array}\right] \in \mathbb{R}^{n \times 2 n}$ describing the natural projection $\pi_{\lambda}: \mathcal{X} \times \mathbb{R}^{n} \rightarrow \mathbb{R}^{n}$ on the $\lambda$-coordinates.

Proof: To begin with, note that, provided the first $k-1$ equations in (10) are satisfied, the $k$-th becomes

$$
\begin{aligned}
0= & \left(\nabla_{z} \operatorname{ad}_{X_{\mathbf{H}}}^{k-1} p\left(z_{0}\right)\right) X_{\mathbf{H}}\left(z_{0}\right)-\left(\nabla_{z} X_{H}(z)\right)^{\top} \operatorname{ad}_{X_{\mathbf{H}}}^{k-1} p\left(z_{0}\right) \\
& -\left(\nabla_{z} \operatorname{ad}_{X_{\mathbf{H}}}^{k-1} z_{0}\right) X_{\mathbf{H}}\left(z_{0}\right)+\left(\nabla_{z} X_{H}(z)\right)^{\top} \operatorname{ad}_{X_{\mathbf{H}}}^{k-1} \operatorname{id}\left(z_{0}\right) \\
= & \left(\nabla_{z} \operatorname{ad}_{X_{\mathbf{H}}}^{k-1} p\left(z_{0}\right)-\nabla_{z} \operatorname{ad}_{X_{\mathbf{H}}}^{k-1} z_{0}\right) X_{\mathbf{H}}\left(z_{0}\right) \\
= & \nabla_{z}\left(\operatorname{ad}_{X_{\mathbf{H}}}^{k-1} p\left(z_{0}\right)-\operatorname{ad}_{X_{\mathbf{H}}}^{k-1} z_{0}\right) X_{\mathbf{H}}\left(z_{0}\right) .
\end{aligned}
$$

Then it can be shown by induction that

$$
\begin{aligned}
& \nabla_{z}\left(\operatorname{ad}_{X_{\mathbf{H}}}^{k-1} p\left(z_{0}\right)-\operatorname{ad}_{X_{\mathbf{H}}}^{k-1} z_{0}\right) X_{\mathbf{H}}\left(z_{0}\right)=-\mathcal{D}_{k-1}\left(X_{\mathbf{H}}\left(z_{0}\right)\right) \\
& +\left[\begin{array}{c}
M \mathcal{D}_{k-1}\left(X_{\mathbf{H}}\left(z_{0}\right)\right) \\
h_{k}\left(z_{0}\right)
\end{array}\right],
\end{aligned}
$$

for any $k \geqslant 1$, with $M=\left[\begin{array}{ll}I_{n} & 0\end{array}\right] \in \mathbb{R}^{n \times 2 n}$. In fact, for $k=1$, one has

$$
\begin{aligned}
0= & {\left[X_{\mathbf{H}}, p\right]\left(z_{0}\right)-\left[X_{\mathbf{H}}, \mathrm{id}\right]\left(z_{0}\right) } \\
= & \left.\left(\left[\begin{array}{c}
M \\
\nabla h(z)
\end{array}\right] X_{\mathbf{H}}(z)-\left(\nabla X_{H}(z)\right)^{\top}\left[\begin{array}{c}
M z \\
h(z)
\end{array}\right]\right)\right|_{z=z_{0}} \\
& -\left.\left(X_{\mathbf{H}}(z)-\left(\nabla X_{\mathbf{H}}(z)\right)^{\top} z\right)\right|_{z=z_{0}} \\
= & {\left[\begin{array}{c}
M X_{\mathbf{H}}\left(x_{0}, \lambda_{0}\right) \\
\lambda_{1}
\end{array}\right]-X_{\mathbf{H}}\left(x_{0}, \lambda_{0}\right), }
\end{aligned}
$$

where the last equality is obtained by noting that $M z_{0}=x_{0}$ and $h\left(z_{0}\right)=\lambda_{0}$. Then, assuming that (19) holds up to a certain index $k$, it can be shown that, for $k+1$,

$$
\begin{aligned}
& \nabla_{z}\left(\operatorname{ad}_{X_{\mathbf{H}}}^{k+1} p(z)-\operatorname{ad}_{X_{\mathbf{H}}}^{k+1} z\right) X_{\mathbf{H}}(z)= \\
& =\nabla_{z}\left(\left[X_{\mathbf{H}}, \operatorname{ad}_{X_{\mathbf{H}}}^{k} p(z)\right]-\left[X_{\mathbf{H}}, \operatorname{ad}_{X_{\mathbf{H}}}^{k} z\right]\right) X_{\mathbf{H}}(z)= \\
& =\nabla_{z}\left(\nabla_{z}\left(\operatorname{ad}_{X_{\mathbf{H}}}^{k} p(z)-\operatorname{ad}_{X_{\mathbf{H}}}^{k} z\right) X_{\mathbf{H}}(z)\right. \\
& \quad+\nabla_{z} X_{\mathbf{H}} \underbrace{\left(\operatorname{ad}_{X_{\mathbf{H}}}^{k} p(z)-\operatorname{ad}_{X_{\mathbf{H}}}^{k} z\right)}_{=0, \text { for } z=z_{0}}) X_{\mathbf{H}}(z),
\end{aligned}
$$

where the property that the previous $k$ equations are satisfied at $z=z_{0}$ has been employed. Therefore,

$$
\begin{aligned}
& \nabla_{z}\left(\operatorname{ad}_{X_{\mathbf{H}}}^{k+1} p\left(z_{0}\right)-\operatorname{ad}_{X_{\mathbf{H}}}^{k+1} z_{0}\right) X_{\mathbf{H}}\left(z_{0}\right)= \\
& =\nabla_{z}\left(\left[\begin{array}{c}
M \mathcal{D}_{k-1}\left(X_{\mathbf{H}}\left(z_{0}\right)\right) \\
h_{k}\left(z_{0}\right)
\end{array}\right]-\mathcal{D}_{k-1}\left(X_{\mathbf{H}}\left(z_{0}\right)\right)\right) X_{\mathbf{H}}\left(z_{0}\right)= \\
& =\left[\begin{array}{c}
M \mathcal{D}_{k}\left(X_{\mathbf{H}}\left(z_{0}\right)\right) \\
h_{k+1}\left(z_{0}\right)
\end{array}\right]-\mathcal{D}_{k}\left(X_{\mathbf{H}}\left(z_{0}\right)\right),
\end{aligned}
$$

where the first equality is obtained by the inductive hypothesis. As a straight-forward consequence of (21), it follows that $\lambda_{i}=\pi_{\lambda} \circ \mathcal{D}_{i-1}\left(X_{\mathbf{H}}\left(z_{0}\right)\right)$ and the claim, hence equation (17), is therefore shown by noting that the values $\lambda_{i}$ describe the coefficients of the Taylor series of $\lambda_{a}(t)$ at $t=0$ and by imposing the terminal condition $\lambda_{a}(T)=0$.

Remark 3: Equations identical to (10), and consequently to (18), can be obtained, in a more convoluted way, by directly considering the subsequent time derivatives of the function $\omega$ 


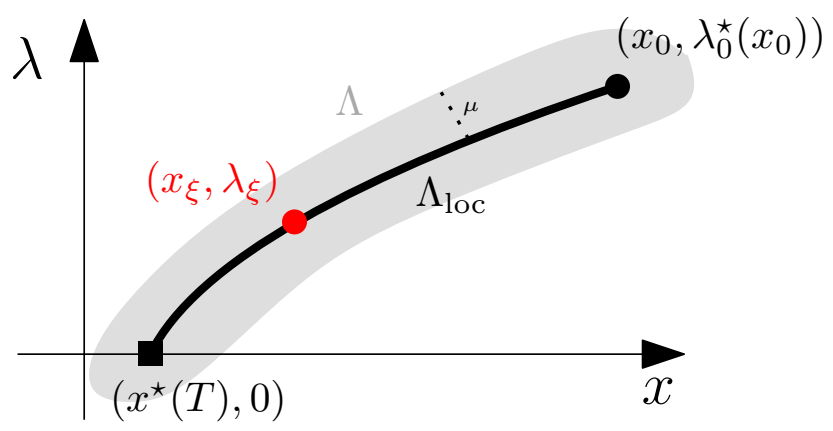

Fig. 2. Graphical illustration of the sets $\Lambda_{\text {loc }}$ (solid black line) and $\Lambda$ (gray region) involved in the proof of Theorem 4.

introduced in (7). For instance, for $k=1$,

$$
\begin{aligned}
\dot{\omega}(t)= & -\frac{\partial}{\partial t} \varphi_{X_{\mathbf{H}}}\left(-t ; M \varphi_{X_{\mathbf{H}}}\left(t ; x_{0}, \lambda_{0}\right), \lambda_{a}(t)\right) \\
& +\nabla_{s} \varphi_{X_{\mathbf{H}}}(-t ; s)\left[\begin{array}{c}
M \frac{\partial}{\partial t} \varphi_{X_{\mathbf{H}}}\left(t ; x_{0}, \lambda_{0}\right) \\
\dot{\lambda}_{a}(t)
\end{array}\right]
\end{aligned}
$$

which coincides with equation (20) for $t=0$ recalling that $\nabla_{s} \varphi_{X_{\mathbf{H}}}(0 ; s)=I_{n}$ and letting $\lambda_{1}:=\dot{\lambda}_{a}(0)$.

Before stating the following result we provide a definition. Given a vector-valued function $\sigma: \mathbb{R}^{n} \rightarrow \mathbb{R}^{n}, \mathbb{V}(\sigma)$ denotes the algebraic set associated to $\sigma$, namely the set $\left\{x \in \mathbb{R}^{n}\right.$ : $\sigma(x)=0\}$. Moreover, given a set $\mathcal{W}$ and a point $v, \mathbf{d}(\mathcal{W}, v)$ denotes the distance between a set and a point, namely

$$
\mathbf{d}(\mathcal{W}, v)=\inf _{w \in \mathcal{W}} d(w, v)
$$

Theorem 4: Consider the finite-horizon optimal control problem (1). Let $x_{0} \in \mathcal{X}$ and $T \in \mathbb{R}_{>0}$ be given and suppose that Assumptions 1-4 hold. Let

$$
\mathcal{T}_{\kappa}\left(\lambda_{0}\right):=\lambda_{0}+N \sum_{i=1}^{\kappa} \mathcal{D}_{i-1}\left(X_{\mathbf{H}}\left(x_{0}, \lambda_{0}\right)\right) \frac{T^{i}}{i !}=0 .
$$

Then for any $\varepsilon>0$ there exist $\kappa(\varepsilon)$ and a compact set $\Lambda \subset R^{n}$ containing $\nabla_{x} V^{\star}\left(0, x_{0}\right)$ such that

$$
\mathbf{d}\left(\mathcal{L}, \nabla_{x} V^{\star}\left(0, x_{0}\right)\right)<\varepsilon,
$$

with $\mathcal{L}:=\mathbb{V}\left(\mathcal{T}_{\kappa}\right) \cap \Lambda$.

Proof: The claim is proved by showing that the difference between $\mathcal{T}_{k}$ and $\mathcal{T}$ can be made arbitrarily small in a compact set containing $\lambda_{0}^{\star}$. Since by Assumptions 2 and 3 the optimal costate $\lambda^{\star}: \mathbb{R} \rightarrow \mathbb{R}^{n}$ is an analytic function at $t=0$ such that the terminal time $T$ belongs to the interior of the domain of convergence with the property that $\mathbb{B}(0,2 T) \subset \mathbb{B}\left(0, r_{H}\right)$, it follows that the Taylor series expansion in the left-hand side of (17) is absolutely, uniformly, convergent to $\lambda^{\star}(T)$. As a consequence, by applying the Cauchy's integral formula to the complex extension of the analytic function $\lambda^{\star}(\cdot)$, which is well-defined and analytic, and since, by $(17), \lambda^{(k)}(0)=$
$N \mathcal{D}_{k}\left(X_{\mathbf{H}}\left(x_{0}, \lambda_{0}\right)\right)$, it follows that

$$
\begin{aligned}
\left|N \mathcal{D}_{k}\left(X_{\mathbf{H}}(x(s), \lambda(s))\right)\right| & \leqslant \frac{k !}{2 \pi} \int_{\partial \overline{\mathbb{B}}(s, \varrho)} \frac{\left|\lambda^{\star}(w)\right|}{|w-s|^{k+1}}|d w| \\
& <c_{\lambda} \frac{k !}{2 \pi} \frac{1}{\varrho^{k+1}} 2 \pi \varrho=c_{\lambda} \frac{k !}{\varrho^{k}},
\end{aligned}
$$

recalling that $\overline{\mathbb{B}}(s, \varrho) \subset \mathbb{B}\left(0, r_{H}\right)$, for some $\varrho>T$ and for any $s \in[0, T]$, where $c_{\lambda}>\max _{w \in \overline{\mathbb{B}}\left(0, r_{H}\right)}\left|\lambda^{\star}(w)\right|$. Therefore,

$$
\left|N \mathcal{D}_{k}\left(X_{\mathbf{H}}(\hat{x}, \hat{\lambda})\right)\right|<c_{\lambda} \frac{k !}{\varrho^{k}},
$$

for any $k \in \mathbb{N}$ and for all $(\hat{x}, \hat{\lambda})$ in the set $\Lambda_{\text {loc }}:=$ $\left\{(\hat{x}, \hat{\lambda}) \in \mathbb{R}^{2 n}: \exists s \in[0, T],(\hat{x}, \hat{\lambda})=\varphi_{X_{\mathbf{H}}}\left(s ; x_{0}, \lambda_{0}^{\star}\left(x_{0}\right)\right)\right\}$. By considering now the Lagrange form of the remainder of the Taylor series expansion $\mathcal{T}_{\kappa}$ with respect to $\mathcal{T}$, it follows that the latter remainder in $\Lambda_{\text {loc }}$ is given by

$$
\mathcal{R}_{k}(s):=N \mathcal{D}_{\kappa+1}\left(X_{\mathbf{H}}\left(x_{\xi}, \lambda_{\xi}\right)\right) \frac{T^{\kappa+1}}{(\kappa+1) !},
$$

for any $s \in[0, T]$ and for some $\left(x_{\xi}, \lambda_{\xi}\right) \in \Lambda_{\text {loc }}$, by Taylor's theorem, hence such that $\left|\mathcal{T}_{\kappa}(\lambda)-\mathcal{T}(\lambda)\right|<$ $\left|N \mathcal{D}_{\kappa+1}\left(X_{\mathbf{H}}\left(x_{\xi}, \lambda_{\xi}\right)\right)\left(T^{\kappa+1}\right)((\kappa+1) !)\right|<c_{\lambda} T^{k+1} / \varrho^{k+1}$, for any $\lambda \in \Lambda_{\text {loc }}$. Thus, by smoothness of the Hamiltonian vector field $X_{\mathbf{H}}$ with respect to $(x, \lambda)$, there exists $\mu>0$ such that

$$
\left|\mathcal{T}_{\kappa}(\lambda)-\mathcal{T}(\lambda)\right|<c_{\lambda}\left(\frac{T}{\varrho}\right)^{\kappa+1}
$$

for all $\lambda \in \Lambda=\Lambda_{\text {loc }}+\mathbb{B}\left(\Lambda_{\text {loc }}, \mu\right)$. The right-hand side of (29) can be then made arbitrarily small by increasing $\kappa$ and recalling that $T<\varrho$.

The intuition behind the statement of Theorem 4 is provided in the following discussion. By relying on Assumption $2, \mathbb{V}(\mathcal{T})$ consists of a single point, namely $\lambda_{0}^{\star}\left(x_{0}\right)=$ $\nabla_{x} V^{\star}\left(0, x_{0}\right)$. The truncation $\mathcal{T}_{\kappa}$ for finite $\kappa$ may instead have multiple (real) roots such that $\mathcal{T}_{\kappa}=0$. The conclusions of Theorem 4 guarantee that among such real solutions there is one that approaches $\lambda_{0}^{\star}\left(x_{0}\right)$ for increasing values of $\kappa$.

Note that determining roots of $\mathcal{T}_{\kappa}\left(\lambda_{0}\right)$ coincides with computing solutions to the system (10) for $k=1, \ldots, \kappa$, with

$$
\lambda_{\kappa}^{\kappa}=-\frac{\kappa !}{T^{\kappa}}\left(\lambda_{0}^{\kappa}+\lambda_{1}^{\kappa} T+\ldots+\lambda_{\kappa-1}^{\kappa}\left(T^{\kappa-1} /(\kappa-1) !\right)\right),
$$

where the superscript $\kappa$ in the unknown vectors $\lambda_{i}^{\kappa}$ hints at the finite truncation of (10).

Remark 4: In addition to the value of $\lambda_{0}^{\kappa}$ that approximates $\nabla_{x} V^{\star}\left(0, x_{0}\right)$ arbitrarily close, the statement of Theorem 4 entails that, instead of using such a value as the (approximate) initial condition of the unstable dynamics (4), the values $\lambda_{i}^{\kappa}$ can be employed to compute a Taylor series expansion for $\lambda_{a}(t)$ as $\tilde{\lambda}(t)=\sum_{k=0}^{\kappa} \lambda_{k}\left(t^{k} / k !\right)$ and then implementing directly the feedforward control law $\tilde{u}(t)=-g(x(t))^{\top} \tilde{\lambda}(t)$. 


\section{INSIGHTS ON THE LINEAR CASE}

The objective of this section consists in specializing the above results to the case of linear systems and quadratic cost functionals, namely

$$
\begin{aligned}
\min _{u} J_{x_{0}}(u) & :=\frac{1}{2} \int_{0}^{T}\left(x(\tau)^{\top} Q x(\tau)+\|u(\tau)\|^{2}\right) d \tau, \\
\text { s.t. } \dot{x}(t) & =A x(t)+B u(t), \quad x(0)=x_{0},
\end{aligned}
$$

with $Q \succeq 0$. Under these structural assumptions the Hamiltonian dynamics are linear and described by the vector-field

$$
X_{\mathbf{H}}=\left[\begin{array}{rr}
A & -B B^{\top} \\
-Q & -A^{\top}
\end{array}\right]\left[\begin{array}{l}
x \\
\lambda
\end{array}\right]:=H\left[\begin{array}{l}
x \\
\lambda
\end{array}\right],
$$

with $H \in \mathbb{R}^{2 n \times 2 n}$ referred to as the Hamiltonian matrix. In the context of Linear-Quadratic (LQ) optimal control, the computation of the optimal solution hinges upon the Differential Riccati Equation (DRE)

$$
-\dot{P}(t)=Q+A^{\top} P(t)+P(t) A-P(t) B B^{\top} P(t),
$$

$P(t)=P(t)^{\top} \in \mathbb{R}^{n \times n}$, with terminal boundary condition $P(T)=0$, which is the linear counterpart of the HJB equation (6). The flow of the former Hamiltonian system then becomes $\varphi_{X_{\mathbf{H}}}\left(t ; z_{0}\right)=e^{H t} z_{0}$. Therefore, the function $\omega(t)$ introduced in (7) reduces, in the linear case, to

$$
\omega(t)=e^{-H t}\left[\begin{array}{c}
M e^{H t}\left[\begin{array}{c}
x_{0} \\
\lambda_{0}
\end{array}\right] \\
\lambda_{a}(t)
\end{array}\right] .
$$

As a result, a claim similar to that given in Theorem 4 in the nonlinear setting can be stated.

Proposition 1: Consider the finite-horizon optimal control problem (31). Let $x_{0} \in \mathcal{X}$ be given. Then for any $\varepsilon>0$ there exists $\kappa(\varepsilon)$ such that the solution $\lambda_{0}^{\kappa}$ to

$$
\left\{\begin{array}{l}
H^{i}\left[\begin{array}{l}
x_{0} \\
\lambda_{0}
\end{array}\right]=\left[\begin{array}{c}
\left.M H^{i}\left[\begin{array}{l}
x_{0} \\
\lambda_{0}
\end{array}\right]\right], i=1, \ldots, \kappa-1 \\
\lambda_{i}
\end{array}\right] \\
H^{\kappa}\left[\begin{array}{c}
x_{0} \\
\lambda_{0}
\end{array}\right]=\left[\begin{array}{c}
M H^{\kappa}\left[\begin{array}{c}
x_{0} \\
\lambda_{0}
\end{array}\right] \\
-\frac{\kappa !}{T^{\kappa}} \sum_{j=0}^{\kappa-1} \lambda_{j}\left(T^{j} / j !\right)
\end{array}\right]
\end{array}\right.
$$

satisfies

$$
\left\|\lambda_{0}^{\kappa}-P(0) x_{0}\right\|_{2}^{2}<\varepsilon
$$

Proof: To begin with it is shown that a truncation of the equations (10) to the order $\kappa$ reduces to (35) in the linear case. This is achieved by imposing that $\omega(t)=\left(x_{0}, \lambda_{0}\right)$ for any $t \geqslant 0$. Note that the latter condition is equivalent to requiring that

$$
\varpi(t):=\left[\begin{array}{c}
M e^{H t}\left[\begin{array}{c}
x_{0} \\
\lambda_{0}
\end{array}\right] \\
\lambda_{a}(t)
\end{array}\right]-e^{H t}\left[\begin{array}{c}
x_{0} \\
\lambda_{0}
\end{array}\right]=0,
$$

for all $t \geqslant 0$. This can be obtained by letting $\varpi^{(i)}(0)=0$ for $i \in \mathbb{N}$, or approximated via a finite truncation with $i=1, \ldots, \kappa$. Therefore,

$$
\varpi^{(i)}(t):=\left[\begin{array}{c}
M H^{i} e^{H t}\left[\begin{array}{c}
x_{0} \\
\lambda_{0}
\end{array}\right] \\
\lambda_{a}^{(i)}(t)
\end{array}\right]-H^{i} e^{H t}\left[\begin{array}{c}
x_{0} \\
\lambda_{0}
\end{array}\right],
$$

which yields (35) together with the constraint $\sum_{i=0}^{\kappa} \lambda_{a}^{(i)}(0)\left(T^{i} / i !\right)=0$. Finally, the inequality (36) is obtained by noting that (35) is a square linear system of equations admitting a unique solution $\lambda_{0}^{\kappa}$.

Remark 5: In the case of LQ optimal control problems over finite-horizon, defining and partitioning the transition matrix associated to the Hamiltonian dynamics (32) according to

$$
e^{H t}:=\Phi_{H}(T, 0)=\left[\begin{array}{ll}
\Phi_{11}(T, 0) & \Phi_{12}(T, 0) \\
\Phi_{21}(T, 0) & \Phi_{22}(T, 0)
\end{array}\right],
$$

yields that $\lambda_{0}^{\star}\left(x_{0}\right)=-\Phi_{22}(T, 0)^{-1} \Phi_{21}(T, 0) x_{0}$, provided $\operatorname{det}\left(\Phi_{22}(T, 0)\right) \neq 0$.

The following statement provides an insight on the structure and sparsity of the system of equations (35) by establishing a relation with the construction mentioned in Remark 5. To provide a concise statement, consider the partition

$$
H^{i}:=\left[\begin{array}{cc}
U_{11, i} & U_{12, i} \\
U_{21, i} & U_{22, i}
\end{array}\right] .
$$

Proposition 2: Consider the finite-horizon optimal control problem (31). Let $x_{0} \in \mathcal{X}$ be given. Then the solution $\lambda_{0}^{\kappa}$ of (35) is given by

$$
\lambda_{0}^{\kappa}=-\left(\sum_{i=0}^{\kappa} U_{22, i}^{i}\left(T^{i} / i !\right)\right)^{-1}\left(\sum_{j=1}^{\kappa} U_{21, j}\left(T^{j} / j !\right)\right) x_{0}
$$

provided the terminal time $T>0$ is such that $\sum_{i=0}^{\kappa} U_{22, i}^{i}\left(T^{i} / i !\right)$ is non-singular.

Proof: To begin with, considering the partition in (39) and noting that $M H^{i}=\left[\begin{array}{ll}U_{11, i} & U_{12, i}\end{array}\right]$, note that the $i$-th equation in (35) can be written as

$$
\left[\begin{array}{c}
U_{11, i} x_{0}+U_{12, i} \lambda_{0} \\
\lambda_{i}
\end{array}\right]=\left[\begin{array}{c}
U_{11, i} \\
U_{21, i}
\end{array}\right] x_{0}+\left[\begin{array}{c}
U_{12, i} \\
U_{22, i}
\end{array}\right] \lambda_{0},
$$

hence

$$
\left[\begin{array}{c}
0 \\
\lambda_{i}
\end{array}\right]=\left[\begin{array}{c}
0 \\
U_{21, i}
\end{array}\right] x_{0}+\left[\begin{array}{c}
0 \\
U_{22, i}
\end{array}\right] \lambda_{0},
$$

which can be then compactly arranged, for $i=1, \ldots, \kappa$, as

$$
\left[\begin{array}{ccccc}
-U_{22,1} & I & 0 & \ldots & 0 \\
-U_{22,2} & 0 & I & \ldots & 0 \\
\vdots & & & \ddots & \vdots \\
G_{1} & & \ldots & & G_{\kappa}
\end{array}\right]\left[\begin{array}{c}
\lambda_{0} \\
\lambda_{1} \\
\vdots \\
\lambda_{k-1}
\end{array}\right]=\left[\begin{array}{c}
U_{21,1} \\
U_{21,2} \\
\vdots \\
U_{21, \kappa}
\end{array}\right] x_{0},
$$




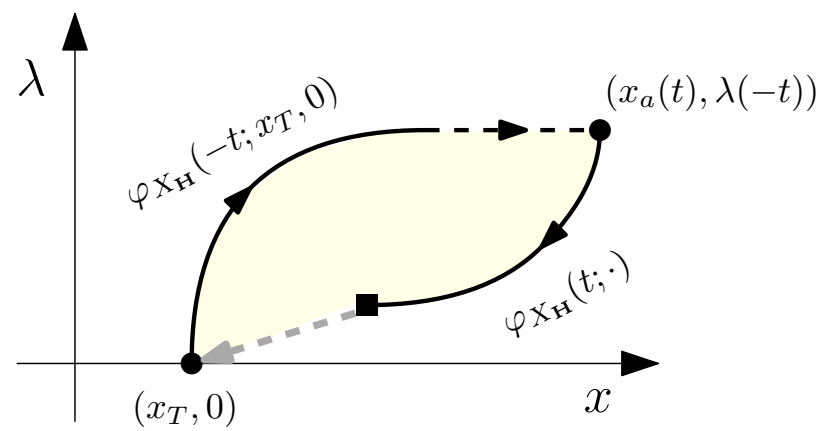

Fig. 3. Graphical representation of the dual composition of the backward and forward flows of the Hamiltonian dynamics (4). The gray dashed vector describes the distance of $\left(\boldsymbol{x}_{T}, \mathbf{0}\right)$ from being a fixedpoint of the composition in (42).

with $G_{1}=-\left(U_{22, \kappa}+\left(T^{\kappa} \kappa !\right) I\right)$ and $G_{j}=-\left(\frac{\kappa !}{j !} T^{j-\kappa}\right) I$, for $j=2, \ldots, \kappa-1$. The claim is then proven by noting that $\lambda_{i}=U_{22, i} \lambda_{0}+U_{21, i} x_{0}, i=1, \ldots, \kappa-1$, and replacing the latter into the last equation of (41).

\section{THE DUAL CASE: FIXED-POINT CHARACTERIZATION OF THE OPTIMAL FINAL STATE}

The fixed-point characterization of the optimal costate discussed in Theorem 1 relies on the property that the initial condition of the entire Hamiltonian dynamics (4) is only partially known, since $x(0)=x_{0}$ is fixed, while the value of the costate $\lambda$ at time $t=0$ must be determined. Such a consideration then leads to the characterization of the optimal costate as pursed in (7), which relies on the composition of Hamiltonian flows.

Interestingly, a somewhat dual strategy is also viable to directly compute the optimal final state by relying on the knowledge of the value of the optimal costate at the terminal time $t=T$, namely $\lambda(T)=0$, and exploiting ideas fully similar to the composition in (7). This aspect is discussed in the following statement, the proof of which is a straightforward adaptation of that of Theorem 1, hence it is omitted.

Theorem 5: Consider the finite-horizon optimal control problem (1). Let $x_{0} \in \mathcal{X}$ and $T \in \mathbb{R}_{>0}$ be given and suppose that Assumptions 1-3 hold. Then a vector $x_{T} \in \mathcal{X}$ is such that $x^{\star}(T)=x_{T}$ if and only if there exists a function $x_{a}$ : $[-T, 0] \rightarrow \mathcal{X}$, analytic at $t=0$, with radius of convergence larger than $T$ and such that $x_{a}(-T)=x_{0}$, with the property that

$$
\omega_{d}(t):=\varphi_{X_{\mathbf{H}}}\left(t ; x_{a}(t), \pi_{\lambda} \circ \varphi_{X_{\mathbf{H}}}\left(-t ; x_{T}, 0\right)\right)=\left(x_{T}, 0\right),
$$

for all $t \in[0, T]$.

By building on the fixed-point characterization (42) in Theorem 5, results identical to those in Section III can be stated and derived. Herein, an in-depth discussion is carried out explicitly in the somewhat explanatory case of linear systems, which allows to highlight the main differences with respect to the results above without any additional burden of notation.
To this end, similarly to (37), let

$$
\varpi_{d}(t):=\left[\begin{array}{c}
x_{a}(t) \\
N e^{-H t}\left[\begin{array}{c}
x_{T} \\
0
\end{array}\right]
\end{array}\right]-e^{-H t}\left[\begin{array}{c}
x_{T} \\
0
\end{array}\right],
$$

with $N=\left[\begin{array}{cc}0 & I_{n}\end{array}\right] \in \mathbb{R}^{n \times 2 n}$, which is equal to zero for any $t$ if and only if $\omega_{d}(t)$ is equal to $\left(x_{T}, 0\right)$ for any $t$, as prescribed by (42) specialized to the linear case.

Proposition 3: Consider the finite-horizon optimal control problem (31). Let $x_{0} \in \mathcal{X}$ be given. Then for any $\varepsilon>0$ there exists $\kappa(\varepsilon)$ such that the solution $x_{T}^{\kappa}$ to

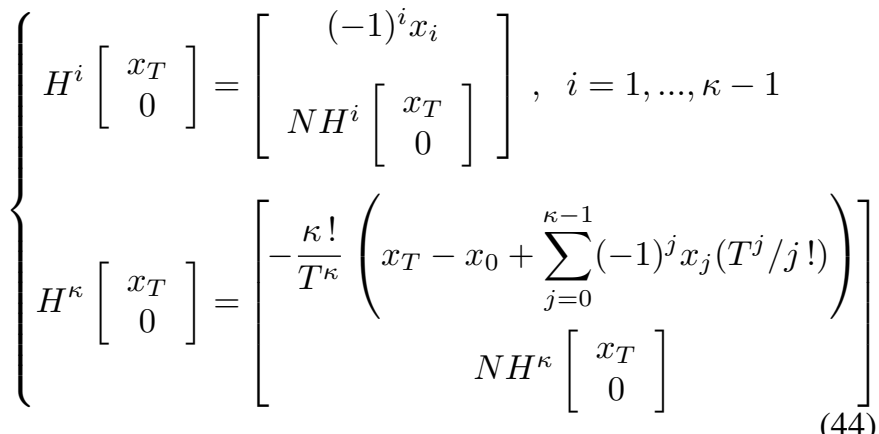

satisfies

$$
\left\|x_{T}^{\kappa}-x^{\star}(T)\right\|_{2}^{2}<\varepsilon
$$

$\diamond$

Proof: By following arguments identical to those employed in the proof of Proposition 1, consider subsequent timederivatives up to the order $\kappa$ of $\varpi_{d}$ at $t=0$, which immediately yields the first $\kappa-1$ equations in (44) where $x_{i}$ denotes the $i$-th derivative of $x_{a}(t)$ evaluated at $t=0$. The last equation, instead, is obtained by additionally considering the constraint $x_{a}(-T)=x_{0}$.

Remark 6: By extending the discussion in Remark 4 to the dual case of the fixed-point characterization of the optimal final state, it may be possible to employ the vectors $x_{i} \in$ $\mathbb{R}^{n}$, solutions to (44), to define a truncated Taylor series approximation of $x_{a}(t)$ according to

$$
\tilde{x}(t)=x_{T}+x_{1}(t-T)+x_{2} \frac{(t-T)^{2}}{2}+\ldots+x_{\kappa} \frac{(t-T)^{\kappa}}{\kappa !},
$$

where $x_{\kappa}=-\frac{\kappa !}{T^{\kappa}}\left(x_{T}-x_{0}+\sum_{j=0}^{\kappa-1}(-1)^{j} x_{j}\left(T^{j} / j !\right)\right)$.

\section{FURTHER DISCUSSIONS}

ON COMPUTATIONAL ASPECTS

The objective of this section is to expand the discussion concerning the computational aspects of the strategy introduced above. This is achieved, first, by pointing out drawbacks of the approach, mainly by means of a few numerical simulations, and then by suggesting possible ways of circumventing or mitigating such shortcomings. 


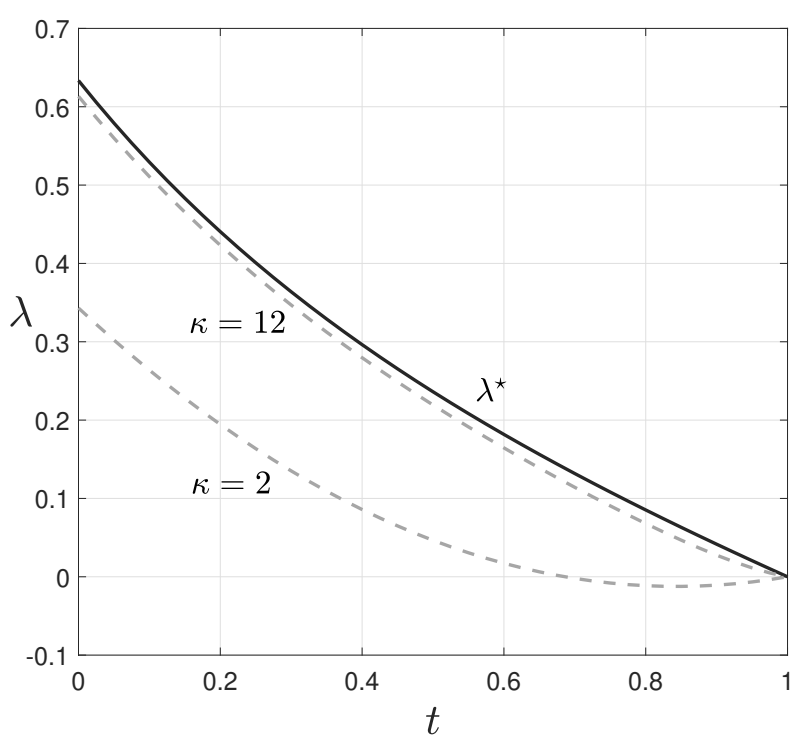

Fig. 4. Time-histories of the optimal costate variable $\lambda^{\star}(t), t \in[0,1]$ (solid black line) and of the approximation obtained via the truncation (24) (solid gray lines), for different values of $\kappa$.

\section{A. Minimizing the time-derivatives of the approximate costate}

By combining the discussion in Remark 4 with the equation (30), it appears that the coefficient $\lambda_{\kappa}^{\kappa}$ alone - which dictates the $\kappa$-th derivative of the function $\tilde{\lambda}(t)$ at time $t=0$ - is "in charge" of compensating for the remainder of the truncation of the Taylor expansion at $t=T$, namely of enforcing $\tilde{\lambda}(T)=0$. This aspect is illustrated via the following numerical simulation.

Example 1: Consider the finite-horizon optimal control problem in (1) with $q(x)=x^{2}, f(x)=x^{2}, g(x)=1$, $x(t) \in \mathbb{R}$, and $T=1$. In this case, the Hamiltonian vector field $X_{\mathbf{H}}$ is given by

$$
X_{\mathbf{H}}(x, \lambda):=\left[\begin{array}{c}
x^{2}-\lambda \\
-x-2 x \lambda
\end{array}\right] .
$$

The latter vector field can be then employed to construct the function $\mathcal{T}_{\kappa}\left(\lambda_{0}\right)$ according to (24) with the recursive operator $\mathcal{D}$ introduced in Definition 1. In this case, then, the function $\mathcal{T}_{\kappa}\left(\lambda_{0}\right)$ is a univariate polynomial in $\lambda_{0}$, for fixed $x_{0} \in \mathbb{R}$. In the following numerical simulations let $x_{0}=0.5$. The time histories of Figure 4 clearly illustrates the discussion at the beginning of this section. In fact, while on one hand the convergence to the optimal value $\lambda_{0}^{\star}$ of a solution to $\mathcal{T}_{\kappa}\left(\lambda_{0}\right)=0$ for increasing $\kappa$ is evident, on the other hand it can be also appreciated that, for small values of $\kappa$, the major shortcoming of the approximate solution $\tilde{\lambda}(t)$ consists in the fact that such a solution - despite being correctly equal to zero at $t=T$ - intersects the value $\lambda=0$ with higher-order derivatives much larger than those of the optimal costate $\lambda^{\star}$, as expected in light of (30).

To counteract the issue illustrated in the example above while maintaining the order $\kappa$ to a reasonable value, it may be possible to introduce and employ correction functions, as detailed below.

Definition 2: A function $\psi_{\lambda}: \mathbb{R} \rightarrow \mathbb{R}$ is said to be an initial correction function of order $\kappa$ if it is such that $\psi_{\lambda}(T)=$ $\psi_{\lambda}^{(1)}(T)=\ldots=\psi_{\lambda}^{(\kappa)}(T)=0$. A possible selection of $\psi_{\lambda}(t)$ is provided by

$$
\psi_{\lambda}(t)=\alpha \frac{(t-T)^{\kappa+1}}{(\kappa+1) !}
$$

with arbitrary $\alpha \in \mathbb{R}^{n}$.

As it appears from Definition 2, the augmented estimate of the costate $\lambda_{\psi}(t)=\tilde{\lambda}(t)+\psi_{\lambda}(t)$ has the property that $\lambda_{\psi}(T)=0$, while the estimate of the initial condition for the state is modified according to $\lambda_{\psi}(0)=\lambda_{0}^{\kappa}+\psi_{\lambda}(0)$. In addition, by resuming the discussion illustrated in the Example 1 above, the criterion on which the arbitrary vector $\alpha$ in (48) is selected is that of minimizing the norm of a specific time-derivative, e.g. the highest one, of the (modified) costate variable in the proximity of the terminal time $T$. More precisely, the choice of $\alpha$ must satisfy

$$
\min _{\alpha} \mathcal{C}_{i}(\alpha, \gamma):=\left|\lambda_{\psi}^{(i)}(\gamma T)-\sum_{j=i+1}^{\kappa} \tilde{\lambda}^{(j)}(0) \frac{(\gamma T)^{j}}{j !}\right|^{2},
$$

for some $\gamma \in \mathbb{R}$, which prescribes the time at which the $i$ th order time derivative of $\lambda_{\psi}$ is minimized. Interestingly, the criterion (49) can be easily minimized in closed form, yielding

$\alpha^{\star}(\gamma):=\arg \min _{\alpha} \mathcal{C}_{i}=-\frac{(\kappa+1-i) !}{((\gamma-1) T)^{\kappa+1-i}} \mathcal{D}_{i-1}\left(X_{\mathbf{H}}\left(x_{0}, \lambda_{0}^{\kappa}\right)\right)$,

where $\lambda_{0}^{\kappa}$ is obtained by solving the equation $\mathcal{T}_{\kappa}(\lambda)=0$. The effect of the selection of the scalar coefficient $\gamma$, which constitutes the remaining degree of freedom, is illustrated by means of the following numerical simulation

Example 2: Consider the finite-horizon optimal control problem in (1) with $q(x)=x^{\top} x, f(x)=A x, g(x)=B$, $x(t) \in \mathbb{R}^{2}$, and $T=2$, where

$$
A=\left[\begin{array}{ll}
0 & 1 \\
0 & 0
\end{array}\right], \quad B=\left[\begin{array}{l}
0 \\
1
\end{array}\right]
$$

namely the system (1b) exhibits double integrator dynamics. Consider then the solution $\lambda_{0}^{4}$ to (35) with $\kappa=4$. Figure 5 depicts the phase plot of the optimal costate (solid black line) and of the approximation $\tilde{\lambda}$ (solid gray line) for $\kappa=4$. Suppose then that an initial correction function $\psi_{\lambda}$ as in (48) is introduced and the vector $\alpha \in \mathbb{R}^{2}$ is selected to minimize $\mathcal{C}_{i}(\alpha, \gamma)$ in (49) for $i=\kappa$, namely to minimize the norm of the highest derivative of $\lambda_{\psi}$ at $t=\gamma T$. By inspecting the structure of the unique minimizer (50), one realizes that the selection of the parameter $\gamma$ naturally leads to a line-search strategy along the direction of the highest derivative defined by the constant vector $\mathcal{D}_{\kappa-1}\left(X_{\mathbf{H}}\left(x_{0}, \lambda_{0}^{\kappa}\right)\right)$, represented by the dotted gray line in Figure 5, which leaves the time history of the costate not altered towards the end of the interval $[0, T]$. The latter points are clearly illustrated by the gray dashed lines in Figure 5 that 


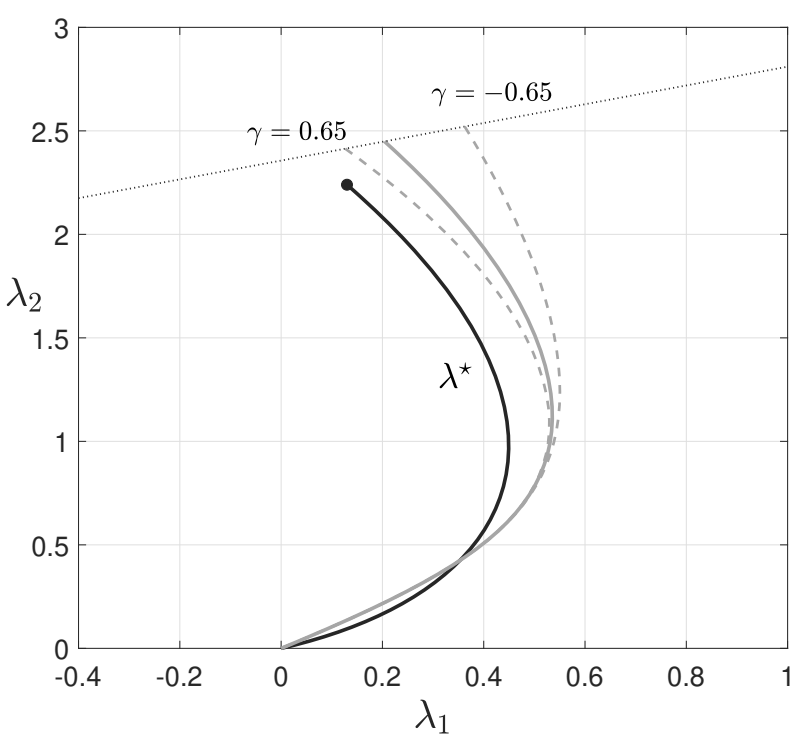

Fig. 5. Phase plot of the optimal costate (solid black line) and of the approximate costate $\tilde{\lambda}$ (solid gray line) obtained with $\kappa=4$ in (35), together with the augmented functions $\boldsymbol{\lambda}_{\psi}$ obtained for different values of the parameter $\gamma$ (dashed gray lines). The dotted line describes the direction of the $\kappa$-th order time derivative of the approximate costate $\tilde{\boldsymbol{\lambda}}$.

describe the augmented function $\lambda_{\psi}$ for different values of the parameter $\gamma$.

Therefore, in general, the choice of $\gamma$ can be based on a linesearch algorithm to determine - along the direction induced by minimizing the norm of a certain derivative of the approximate costate - the augmented estimate

$$
\lambda_{\psi}(0)=\lambda_{0}^{\kappa}+\alpha^{\star}(\gamma) \frac{(-T)^{\kappa+1}}{(\kappa+1) !}
$$

that yields a minimal norm of the costate $\lambda_{\psi}(T)$ at the terminal time. Moreover, the above construction can be iterated by considering the minimization of the norm of different time derivatives of the augmented function $\lambda_{\psi}(t)$, with the sequence of such derivatives induced by the optimal steps $\alpha_{k}^{\star}\left(\gamma_{k}^{\star}\right)$ of the line-search algorithm. This aspect is illustrated by means of two consecutive steps of the algorithm in Figure 6, which completes the discussion of Example 2. Note that the simulation is limited to two steps to more clearly visualize the directions induced by the derivatives of the approximate costate $\tilde{\lambda}$ and that a longer sequence of iterations would converge to the exact value of $\lambda_{0}^{\star}$, as it can be appreciated from Figure 7.

\section{B. Time-scaling}

The statements of Theorems 3 and 4 suggest that large values of the terminal time $T$ may hinder the precision and reliability of the numerical operations involved in the computation of the estimate of the optimal costate. One may then envision a strategy that consists in scaling the time variable $t$ with respect to which the system (1b) is defined in such a way that the finite-horizon problem can be written in the (scaled) time interval $[0,1]$. However, while allowing

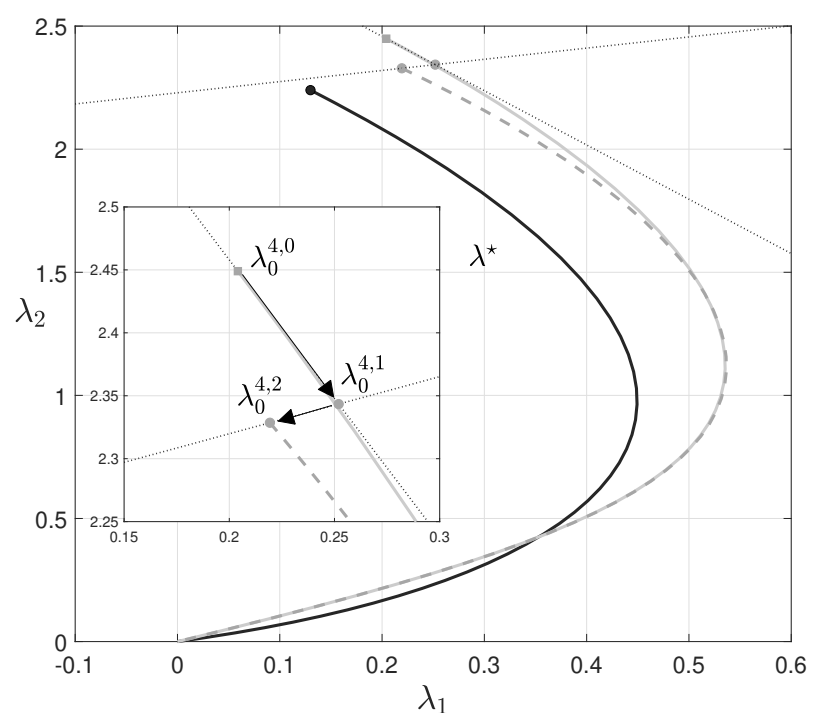

Fig. 6. Phase plot of the optimal costate (solid black line) and of the approximate costate $\tilde{\boldsymbol{\lambda}}$ (solid gray line) obtained with $\kappa=4$ in (35). The gray square depicts $\lambda_{0}^{4}$, while the gray circles represent two steps of the line search algorithm obtained via (49) and the optimal selection of $\gamma$.

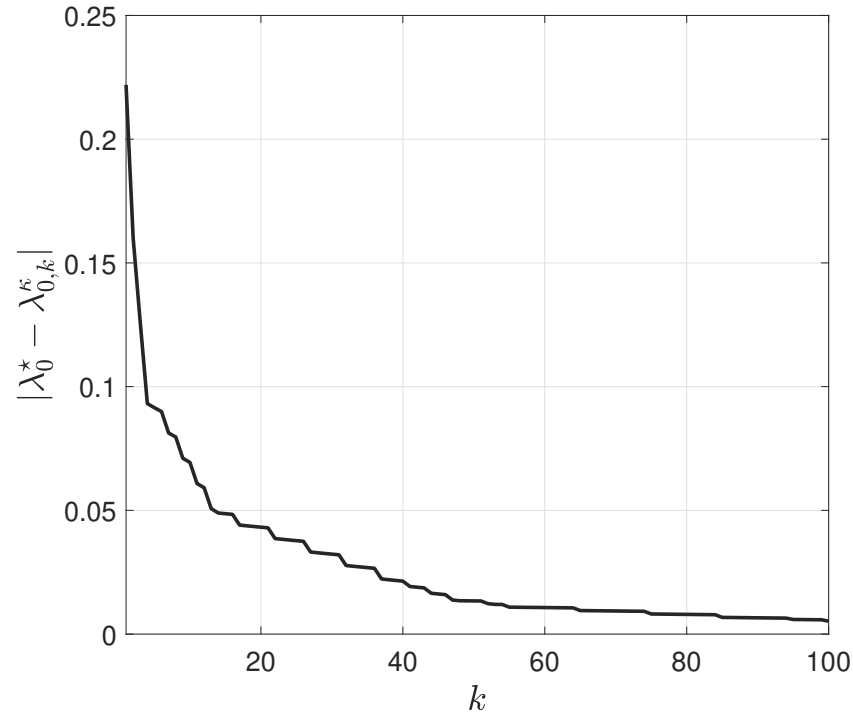

Fig. 7. Convergence of the line-search algorithm to $\lambda_{0}^{\star}$ with respect to the iteration number $\boldsymbol{k}$.

to relax the requirements in Assumption 3 associated to a smaller interval, it can be shown that such an approach leads to numerical computations identical to those of the unscaled case.

To this end, define the scaled time $\tau(t)$ such that $\tau(0)=0$ and $\tau(T)=1$, e.g. consider the linearly scaled time $\tau=t / T$. According to this definition, it is easy to see that the scaled dynamics become

$$
\frac{d x}{d \tau}=\frac{d x}{d t} \frac{d \tau}{d t}=T f(x(\tau))+T g(x(\tau)) u(\tau)
$$


and that the cost functional is similarly scaled as

$$
\frac{1}{2} \int_{0}^{1}\left(T q(x(\tau))+T\|u(\tau)\|^{2}\right) d \tau .
$$

It is then not difficult to see that the scaled Hamiltonian vector field $X_{\mathbf{H}}^{s}(x, \lambda)$ is related to the unscaled one according to $X_{\mathbf{H}}^{s}(x, \lambda)=T X_{\mathbf{H}}(x, \lambda)$. Therefore, to approximate the initial condition of the optimal costate in the setting of the scaled formulation, one should solve the equations

$$
\begin{aligned}
0 & =\lambda_{0}+N \sum_{i=1}^{\kappa} \mathcal{D}_{i-1}\left(X_{\mathbf{H}}^{s}\left(x_{0}, \lambda_{0}\right)\right) \frac{1}{i !} \\
& =\lambda_{0}+N \sum_{i=1}^{\kappa} \mathcal{D}_{i-1}\left(T X_{\mathbf{H}}\left(x_{0}, \lambda_{0}\right)\right) \frac{1}{i !} \\
& =\lambda_{0}+N \sum_{i=1}^{\kappa} T^{i} \mathcal{D}_{i-1}\left(X_{\mathbf{H}}\left(x_{0}, \lambda_{0}\right)\right) \frac{1}{i !},
\end{aligned}
$$

where the last equality is obtained by definition of the operator $\mathcal{D}_{i}$, which coincides with (24). Finally, in the case of nonlinearly scaled time according to $\tau=\sigma(t)$, with $\sigma(0)=0$ and $\sigma(T)=1$, the scaled dynamics becomes time-varying as $d x / d \tau=\dot{\sigma}\left(\sigma^{-1}(\tau)\right)(f(x(\tau))+g(x(\tau)) u(\tau))$.

\section{Minimizing the "gap" between approximate state and costate}

With the aim of combining ideas similar to those introduced in Section VI-A with the dual characterization of the optimal state as in Section V, and in particular in Remark 6, consider the following statement that extends Definition 2.

Definition 3: A function $\psi_{x}: \mathbb{R} \rightarrow \mathbb{R}$ is said to be a terminal correction function of order $\kappa$ if it is such that $\psi_{x}(0)=\psi_{x}^{(1)}(0)=\ldots=\psi_{x}^{(\kappa)}(0)=0$. A possible selection of $\psi_{x}(t)$ is provided by

$$
\psi_{x}(t)=\beta \frac{t^{\kappa+1}}{(\kappa+1) !},
$$

with arbitrary $\beta \in \mathbb{R}^{n}$.

Clearly, the modified approximate state $x_{\psi}(t)=\tilde{x}(t)+$ $\psi_{x}(t)$ still verifies $x_{\psi}(0)=x_{0}$. Along the same lines of what has been discussed above, the initial and terminal correction functions may be employed to provide a more accurate approximation of the optimal state and costate without increasing the complexity of the algebraic equation (24). This objective can be achieved by selecting the parameters $\alpha$ and $\beta$ according to the criterion

$\min _{\alpha, \beta}\left|\left[\begin{array}{c}\dot{\tilde{x}}(\bar{t})+\dot{\psi}_{x}(\bar{t}) \\ \dot{\tilde{\lambda}}(\bar{t})+\dot{\psi}_{\lambda}(\bar{t})\end{array}\right]-X_{\mathbf{H}}\left(\tilde{x}(\bar{t})+\psi_{x}(\bar{t}), \tilde{\lambda}(\bar{t})+\psi_{\lambda}(\bar{t})\right)\right|^{2}$,

namely such that the distance between the actual time derivatives of the approximate state and costate - obtained according to the (distinct) strategies discussed in Sections III and V - and the corresponding value of the Hamiltonian vector field, at a specific time $\bar{t} \in[0, T]$, is minimized.

To conclude this section two notable cases of interest in the linear setting are discussed in detail. These consist in the selection of the available parameters $\alpha$ and $\beta$ to improve, on one hand, the approximation of the costate initial condition obtained by minimizing (57) at $\bar{t}=0$ or, on the other hand, the approximation of the state terminal condition obtained by minimizing (57) at $\bar{t}=T$. Such cases are discussed in the following two statements, respectively.

Proposition 4: Consider the finite-horizon optimal control problem (31). Fix $\kappa>0$ and consider the solutions $\lambda_{0}^{\kappa}, \lambda_{i}$ and $x_{T}, x_{i}$, with $i=1, \ldots, \kappa$, to (35) and (44), respectively. Suppose that $\{(\kappa+1) / T\} \cap \sigma(A)=\emptyset$. Then

$$
\alpha^{\star}:=-\left(G_{2}^{\top} G_{2}+G_{4}^{\top} G_{4}\right)^{-1}\left(G_{2}^{\top} G_{1}+G_{4}^{\top} G_{3}\right)
$$

with

$$
\begin{aligned}
G_{1} & :=\sum_{j=1}^{\kappa} x_{j} \frac{(-T)^{i-1}}{(i-1) !}-A x_{0}+B R^{-1} B^{\top} \lambda_{0}^{\kappa}, \\
G_{2} & :=B R^{-1} B^{\top}(-T)^{\kappa+1} /(\kappa+1) !, \\
G_{3} & :=\lambda_{1}+Q x_{0}+A^{\top} \lambda_{0}^{\kappa}, \\
G_{4} & :=\frac{(-T)^{\kappa}}{\kappa !} I+\frac{(-T)^{\kappa+1}}{(\kappa+1) !} A^{\top},
\end{aligned}
$$

is the unique minimizer of (57) at $\bar{t}=0$.

$\diamond$

Proof: The claim is easily proven by noting that in the case of system (31b) the Hamiltonian vector field $X_{\mathbf{H}}$ is linear and that, by construction $\tilde{x}(0)+\psi_{x}(0)=x_{0}$, $\tilde{\lambda}(0)+\psi_{\lambda}(0)=\lambda_{0}^{\kappa}+\alpha(-T)^{\kappa+1} /(\kappa+1)$ !, while $\dot{\tilde{x}}(0)+$ $\dot{\psi}_{x}(0)=\sum_{i=1}^{\kappa} x_{i}(-T)^{i-1} /(i-1) !$ and $\dot{\tilde{\lambda}}(0)+\dot{\psi}_{\lambda}(0)=\lambda_{1}+$ $\alpha(-T)^{\kappa} /(\kappa !)$. Therefore, the minimization in (57) becomes

$$
\min _{\alpha}\left|\left[\begin{array}{c}
G_{1}+G_{2} \alpha \\
G_{3}+G_{4} \alpha
\end{array}\right]\right|^{2}
$$

which depends only on the vector $\alpha$, and where the matrices $G_{j}, j=1, \ldots, 4$ are defined in (59). The proof is then concluded provided it is shown that the positive semidefinite matrix $G_{2}^{\top} G_{2}+G_{4}^{\top} G_{4}$ is invertible. To this end, note that $G_{4}=(-1)^{\kappa} \frac{T^{\kappa}}{\kappa !}\left(I-\frac{T}{\kappa+1} A^{\top}\right)$ is non-singular by the assumption on the spectrum of the matrix $A$.

A similar result can be stated in the case in which the solution to (57) is employed to improve the approximation of the terminal value of the state $\tilde{x}$, namely at $\bar{t}=T$.

Proposition 5: Consider the finite-horizon optimal control problem (31). Fix $\kappa>0$ and consider the solutions $\lambda_{0}^{\kappa}, \lambda_{i}$ and $x_{T}, x_{i}$, with $i=1, \ldots, \kappa$, to (35) and (44), respectively. Suppose that $\{(\kappa+1) / T\} \cap \sigma(A)=\emptyset$. Then

$$
\beta^{\star}:=-\left(G_{2}^{\top} G_{2}+G_{4}^{\top} G_{4}\right)^{-1}\left(G_{2}^{\top} G_{1}+G_{4}^{\top} G_{3}\right)
$$




\section{REFERENCES}

$$
\begin{aligned}
G_{1} & :=x_{1}-A x_{T}, \\
G_{2} & :=\frac{T^{\kappa}}{\kappa !} I-\frac{T^{\kappa+1}}{(\kappa+1) !} A, \\
G_{3} & :=\sum_{j=1}^{\kappa} \lambda_{j} \frac{T^{i-1}}{(i-1) !}+Q x_{T}, \\
G_{4} & :=\frac{T^{\kappa+1}}{(\kappa+1) !} Q,
\end{aligned}
$$

is the unique minimizer of (57) at $\bar{t}=T$.

The proof of Proposition 5 follows arguments identical to those employed in the proof of Proposition 4, hence it is omitted.

\section{CONCLUSIONS AND FURTHER DISCUSSIONS}

In this paper a fixed-point characterization of the optimal costate variable in finite-horizon optimal control problems has been proposed for linear and nonlinear systems. In particular, the main idea revolves around the property that the optimal initial condition of the Hamiltonian dynamics must verify, together with an auxiliary function that replicates the timeevolution of the costate itself, a fixed-point condition with respect to the composition of a forward and backward flows of the Hamiltonian vector field. Due to the presence of time in the above condition, although rather insightful such a characterization is however not particularly practical and amenable to numerical computations. Therefore, it has been shown that the above property can be cast in more constructive requirements by relying on the notion of (repeated) Lie brackets, which involve the Hamiltonian vector field and a suitably defined projective vector field and which are evaluated at a single point in the state-space. This strategy then yields a system of algebraic equations in the costate initial condition, the number of which always matches the dimension of the state of the underlying system regardless of the prescribed degree of accuracy for the approximation.

As further developments concerning the computation of the optimal costate, it would be of interest to expand the discussion about the main computational features of the algorithm. The final outreach is to envision and develop computationally efficient routines to compute or approximate the optimal costate. Moreover, the idea of deriving algebraic conditions from fixed-point characterizations in composition of flows seems appealing in diverse fields of application, in additional to optimal control, such as the computation of invariant sets, periodic orbits of nonlinear systems as well as concepts related to nonlinear reachability and controllability. Finally, similar abstract, geometric considerations may be extended also to the class of continuous-time stochastic systems by relying on an adapted formulation of the Hamiltonian dynamics, in which the backward evolution must be suitably defined to avoid violating nonanticipativness of the solutions [41].
[1] B. D. Anderson and J. B. Moore, Optimal control: linear quadratic methods. Prentice Hall, New Jersey, 1990. Reprinted by Dover in 2007.

[2] D. P. Bertsekas, Dynamic programming and optimal control. Athena scientific Belmont, MA, 2005.

[3] H. Kwakernaak and R. Sivan, Linear optimal control systems. Wiley, New York, 1972.

[4] D. Liberzon, Calculus of variations and optimal control theory: a concise introduction. Princeton University Press, 2011.

[5] R. Vinter, Optimal control. Springer Science \& Business Media, 2010.

[6] J. C. Doyle, K. Glover, P. P. Khargonekar, and B. A. Francis, "Statespace solutions to standard $\mathrm{H}_{2}$ and $H_{\infty}$ control problems," IEEE Transactions on Automatic Control, vol. 34, no. 8, pp. 831-847, 1989.

[7] A. J. van der Schaft, L2-gain and passivity techniques in nonlinear control. 2nd edition, Springer, 2000.

[8] D. A. Carlson, A. B. Haurie, and A. Leizarowitz, Infinite horizon optimal control: deterministic and stochastic systems. Springer Science \& Business Media, 2012.

[9] R. Bellman, Dynamic Programming. Princeton, NJ, USA: Princeton University Press, 1957.

[10] L. S. Pontryagin, V. G. Boltyanskii, R. V. Gamkrelidze, and E. F. Mishchenko, The Mathematical Theory of Optimal Processes. John Wiley \& sons, New York, 1962.

[11] D. L. Lukes, "Optimal regulation of nonlinear dynamical systems," SIAM Journal on Control, vol. 7, no. 1, pp. 75-100, 1969.

[12] A. Wernli and G. Cook, "Successive control for the nonlinear quadratic regulator problem," Automatica, vol. 11, pp. 75-84, 1975.

[13] M. Falcone, "A numerical approach to the infinite horizon problem of deterministic control theory," Applied Mathematics and Optimization, vol. 15, pp. 1-13, 1987.

[14] F. Camilli, M. Falcone, P. Lanucara, and A. Seghini, "A domain decomposition method for Bellman equation," Contemporary Math., vol. 180, pp. 477-483, 1994.

[15] R. W. Beard, G. N. Saridis, and J. T. Wen, "Galerkin approximations of the generalized Hamilton-Jacobi-Bellman equation," Automatica, vol. 33, no. 12, pp. 2159-2177, 1997.

[16] W. M. McEneaney, "A curse-of-dimensionality-free numerical method for solution of certain HJB PDEs," SIAM Journal on Control and Optimization, vol. 46, no. 4, pp. 1239-1276, 2007.

[17] T. Hunt and A. J. Krener, "Improved patchy solution to the HamiltonJacobi-Bellman equations," in Proc. of the 49th Conference on Decision and Control, Atlanta, 2010.

[18] M. Sassano and A. Astolfi, "Dynamic approximate solutions of the HJ inequality and of the HJB equation for input-affine nonlinear systems," IEEE Transactions on Automatic Control, vol. 57, no. 10, pp. 24902503,2012

[19] M. Bardi and I. Capuzzo-Dolcetta, Optimal Control and Viscosity Solutions to Hamilton-Jacobi-Bellman Equations. Birkhauser, Boston, 1997.

[20] M. G. Crandall and P. L. Lions, "Viscosity solutions of Hamilton-Jacobi equations," Trans. Amer. Math. Soc., vol. 227, pp. 1-42, 1983.

[21] M. A. Patterson and A. V. Rao, "GPOPS-II: A MATLAB software for solving multiple-phase optimal control problems using hp-adaptive gaussian quadrature collocation methods and sparse nonlinear programming," ACM Transactions on Mathematical Software (TOMS), vol. 41, no. 1, pp. (1) 1-37, 2014.

[22] J. T. Betts, "Sparse Jacobian updates in the collocation method for optimal control problems," Journal of Guidance, Control, and Dynamics, vol. 13 , no. 3 , pp. 409-415, 1990.

[23] — "Survey of numerical methods for trajectory optimization," Journal of guidance, control, and dynamics, vol. 21, no. 2, pp. 193-207, 1998.

[24] D. A. Benson, G. T. Huntington, T. P. Thorvaldsen, and A. V. Rao, "Direct trajectory optimization and costate estimation via an orthogonal collocation method," Journal of Guidance, Control, and Dynamics, vol. 29, no. 6, pp. 1435-1440, 2006.

[25] C. L. Darby, D. Garg, and A. V. Rao, "Costate estimation using multipleinterval pseudospectral methods," Journal of spacecraft and rockets, vol. 48, no. 5, pp. 856-866, 2011.

[26] G. Elnagar, M. A. Kazemi, and M. Razzaghi, "The pseudospectral legendre method for discretizing optimal control problems," IEEE transactions on Automatic Control, vol. 40, no. 10, pp. 1793-1796, 1995.

[27] D. Garg, W. W. Hager, and A. V. Rao, "Pseudospectral methods for solving infinite-horizon optimal control problems," Automatica, vol. 47, no. 4 , pp. 829-837, 2011. 
[28] D. Garg, M. Patterson, W. W. Hager, A. V. Rao, D. A. Benson, and G. T. Huntington, "A unified framework for the numerical solution of optimal control problems using pseudospectral methods," Automatica, vol. 46, no. 11, pp. 1843-1851, 2010.

[29] A. V. Rao, D. A. Benson, C. Darby, M. A. Patterson, C. Francolin, I. Sanders, and G. T. Huntington, "Algorithm 902: GPOPS, a MATLAB software for solving multiple-phase optimal control problems using the gauss pseudospectral method," ACM Transactions on Mathematical Software (TOMS), vol. 37, no. 2, pp. (22) 1-39, 2010

[30] O. J. F. Y. Nie and E. C. Kerrigan, "ICLOCS2: Solve your optimal control problems with less pain," in Proc. 6th IFAC Conference on Nonlinear Model Predictive Control, 2018.

[31] S. Jain and P. Tsiotras, "Trajectory optimization using multiresolution techniques," Journal of Guidance, Control, and Dynamics, vol. 31, no. 5, pp. 1424-1436, 2008

[32] Y. Zhao and P. Tsiotras, "Density functions for mesh refinement in numerical optimal control," Journal of guidance, control, and dynamics, vol. 34, no. 1, pp. 271-277, 2011.

[33] C. L. Darby, W. W. Hager, and A. V. Rao, "An hp-adaptive pseudospectral method for solving optimal control problems," Optimal Control Applications and Methods, vol. 32, no. 4, pp. 476-502, 2011.

[34] G. N. Elnagar and M. Razzaghi, "A collocation-type method for linear quadratic optimal control problems," Optimal Control Applications and Methods, vol. 18, no. 3, pp. 227-235, 1997.

[35] S. Kameswaran and L. T. Biegler, "Convergence rates for direct transcription of optimal control problems using collocation at Radau points," Computational Optimization and Applications, vol. 41, no. 1, pp. 81126, 2008.

[36] D. Bertsekas, Nonlinear Programming. Athena Scientific, 2nd ed., 1999.

[37] L. T. Biegler and V. M. Zavala, "Large-scale nonlinear programming using IPOPT: An integrating framework for enterprise-wide dynamic optimization," Computers \& Chemical Engineering, vol. 33, no. 3, pp. 575-582, 2009.

[38] M. Sassano and A. Astolfi, "Combining Pontryagin's Principle and Dynamic Programming for linear and nonlinear systems," IEEE Transactions on Automatic Control, to appear.

[39] D. E. Kirk, Optimal control theory: an introduction. Courier Corporation, 2004.

[40] A. Isidori, Nonlinear control systems. Springer-Verlag, 3rd edition, 1995.

[41] J. Yong and X. Y. Zhou, "Maximum principle and stochastic Hamiltonian systems," in Stochastic Controls. Springer, 1999, pp. 101-156.

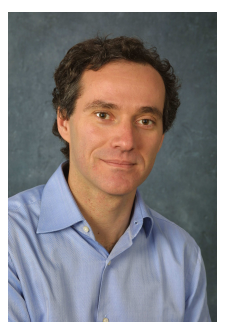

Alessandro Astolfi was born in Rome, Italy, in 1967. He graduated in electrical engineering from the University of Rome in 1991. In 1992 he joined ETH-Zurich where he obtained a M.Sc. in Information Theory in 1995 and the Ph.D. degree with Medal of Honor in 1995 with a thesis on discontinuous stabilization of nonholonomic systems. In 1996 he was awarded a Ph.D. from the University of Rome "La Sapienza" for his work on nonlinear robust control. Since 1996 he has been with the Electrical and Electronic Engineering Department of Imperial College London, London (UK), where he is currently Professor of Nonlinear Control Theory and Head of the Control and Power Group. From 1998 to 2003 he was also an Associate Professor at the Dept. of Electronics and Information of the Politecnico of Milano. Since 2005 he has also been a Professor at Dipartimento di Ingegneria Civile e Ingegneria Informatica, University of Rome Tor Vergata. He has been a visiting lecturer in "Nonlinear Control" in several universities, including ETH-Zurich (1995-1996); Terza University of Rome (1996); Rice University, Houston (1999); Kepler University, Linz (2000); SUPELEC, Paris (2001), Northeastern University (2013).

His research interests are focused on mathematical control theory and control applications, with special emphasis for the problems of discontinuous stabilization, robust and adaptive control, observer design and model reduction. He is the author of more than 150 journal papers, of 30 book chapters and of over 240 papers in refereed conference proceedings. He is the author (with D. Karagiannis and R. Ortega) of the monograph "Nonlinear and Adaptive Control with Applications" (Springer-Verlag).

$\mathrm{He}$ is the recipient of the IEEE CSS A. Ruberti Young Researcher Prize (2007), the IEEE RAS Googol Best New Application Paper Award (2009), the IEEE CSS George S. Axelby Outstanding Paper Award (2012) and the Automatica Best Paper Award (2017). He is a "Distinguished Member" of the IEEE CSS, IEEE Fellow and IFAC Fellow.

He served as Associate Editor for Automatica, Systems and Control Letters, the IEEE Trans. on Automatic Control, the International Journal of Control, the European Journal of Control and the Journal of the Franklin Institute; as Area Editor for the Int. J. of Adaptive Control and Signal Processing; as Senior Editor for the IEEE Trans. on Automatic Control; and as Editor-in-Chief for the European Journal of Control. He is currently Editor-in-Chief of the IEEE Trans. on Automatic Control. He served as Chair of the IEEE CSS Conference Editorial Board (20102017) and in the IPC of several international conferences. He has been/is a Member of the IEEE Fellow Committee (2016), (2019-2020).

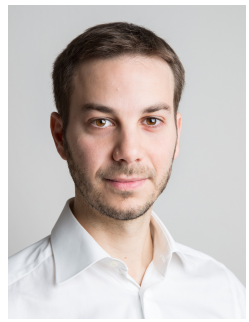

Mario Sassano was born in Rome, Italy, in 1985. He received the B.S degree in Automation Systems Engineering and the M.S degree in Systems and Control Engineering from the University of Rome "La Sapienza", Italy, in 2006 and 2008, respectively. In 2012 he was awarded a Ph.D. degree by Imperial College London, UK, where he had been a Research Assistant in the Department of Electrical and Electronic Engineering (2009-2012). Currently he is an Assistant Professor at the University of Rome "Tor Vergata", Italy. His research interests are focused on nonlinear observer design, optimal control and differential game theory with applications to mechatronical systems and output regulation for hybrid systems. He is Associate Editor of the European Journal of Control and of the IEEE CSS and EUCA Conference Editorial Boards. 